\title{
Deformation of spherical CR structures and the universal Picard variety
}

\author{
JiH-Hsin Cheng AND I-Hsun TSAI ${ }^{1}$
}

\begin{abstract}
We study deformations of a spherical CR circle bundle over a Riemann surface of genus $>1$. Roughly speaking, there is a diffeomorphism between such a deformation space and the unramified universal Picard variety. On the way to the latter, we actually give a differential-geometric proof of the structure and dimension of the unramified universal Picard variety.
\end{abstract}

\section{Introduction.}

In this paper we study the deformation of spherical CR circle bundles over Riemann surfaces of genus $>1$. (for genus $=0$ or 1 , see $[B S]$ for some discussions) We find that there is a one-to-one correspondence between such a deformation space and the so-called universal Picard variety. Let $N$ be a closed (compact without boundary) Riemann surface of genus $g>1$. Let $L$ be a holomorphic line bundle over $N$ with the first Chern class $c_{1}(L)$ (in $\left.H^{2}(N, Z)=Z\right)<0$. The universal Picard variety with given genus $g>1$ and $c_{1}<0$, denoted by $P_{i c}$, is the quotient space of all such pairs $(L, N)$ modulo the equivalence relation given by holomorphic bundle isomorphisms.

First given $(L, N)$, we can find a hermitian metric \|\|$: L \rightarrow R^{+} \cup\{0\}$ such that the circle bundle $S_{L} \subset L$ defined by \|\|$=1$ is spherical relative to the induced CR structure, denoted by $J_{L}$ or $\left(H_{L}, J_{L}\right)$. ( $H_{L}$ is the induced contact bundle)(see section 2 for more details) Now fix $[(\hat{L}, \hat{N})]$ in $P_{i c}$. We have the following convention about the regularity of geometric objects: a geometric object is assumed to be smooth $\left(C^{\infty}\right)$ if we do not specify its regularity. We consider the deformation of spherical CR structures on $\hat{S}=S_{\hat{L}}$. By a theorem of Gray [Gr], we may just fix the underlying contact bundle $\hat{H}=H_{\hat{L}}$ with the orientation induced by $\hat{J}=J_{\hat{L}}$. Let $\widetilde{\mathfrak{S}}$ denote the space of all spherical CR manifolds $(\hat{S}, \hat{H}, J)$ with $J$ oriented and compatible with $\hat{H}$.

\footnotetext{
${ }^{1}$ Research supported in part by National Science Council grants of R.O.C., NSC 86-2115-M-001-003 and NSC 85-2121-M-002-011 respectively.
} 
([CL1]) Let $C_{\hat{H}}$ be the orientation-preserving contact diffeomorphism group relative to $\hat{H}$. $C_{\hat{H}}$ acts on $\widetilde{\mathfrak{S}}$ by pulling back. Let $C_{\hat{H}}^{0}$ denote the identity component of $C_{\hat{H}}$. Define the Teichmuller-type space $\mathfrak{S}^{t}$ to be $\widetilde{\mathfrak{S}} / C_{\hat{H}}^{0}$. Similarly we can describe $P_{i c}$ based on a fixed background line bundle and define the Teichmuller-type space $P_{i c}^{t}$. (see section 3 for details) $P_{i c}^{t}$ can be endowed with a natural complex manifold structure. (see Theorem $\mathrm{C}$ below) The map $\tau:[(L, N)] \rightarrow\left[\left(S_{L}, H_{L}, J_{L}\right)\right]$ (equivalence relation given by diffeomorphisms) gives rise to a map $\tau^{t}: P_{i c_{0}}^{t} \rightarrow \mathfrak{S}_{0}^{t}$. (see section 5 for definitions)

Theorem A. (1) $\mathfrak{S}_{0}^{t}$ has a natural smooth manifold structure with dimension equal to $8 g-6$.

(2) The map $\tau^{t}: P_{i c_{0}}^{t} \rightarrow \mathfrak{S}_{0}^{t}$ is a diffeomorphism.

Theorem A is in the same spirit as that of describing Teichmuller space by conformal classes. It is known in Teichmuller theory that we can pick up a unique hyperbolic metric as a representative for each conformal class. The similar situation occurs for our spherical CR manifolds. Let $\mathfrak{M}_{-1,0}$ denote the quotient space of all pseudohermitian manifolds $(M, H, J, \theta)$ with $(M, H)$ being contact-diffeomorphic to $(\hat{S}, \hat{H})$ so that the (pseudohermitian or Tanaka-Webster) curvature $R_{J, \theta}$ equals -1 and the torsion $A_{J, \theta}$ vanishes modulo the equivalence relation given by diffeomorphisms. ([We1],[Tan]) It follows that $\operatorname{such}(M, H, J)$ is spherical and for $\left(\hat{S}, \hat{H}, \hat{J}=J_{\hat{L}}\right)$ we can always pick up a unique contact form $\hat{\theta}=\theta_{\hat{L}}$ with $R_{\hat{J}, \hat{\theta}}=-1$ and $A_{\hat{J}, \hat{\theta}}=0$. $\hat{H}$ is given a natural orientation by claiming $(v, \hat{J} v)$ is an oriented basis of $\hat{H}$ for any nonzero $v$ in $\hat{H}$. A pseudohermitian structure $(J, \theta)$ on $(\hat{S}, \hat{H})$ is called oriented if both $\mathrm{J}$ and $\theta$ are oriented for $\hat{H}$. ([CL1]) To study $\mathfrak{M}_{-1,0}$ we may just fix $(M, H)=(\hat{S}, \hat{H})$ and consider the space of all oriented pseudohermitian structures $(J, \theta)$ on $(\hat{S}, \hat{H})$ with $R_{J, \theta}=-1, A_{J, \theta}=0$, denoted by $\widetilde{\mathfrak{M}}_{-1,0}$. It is clear that $C_{\hat{H}}$ acts on $\widetilde{\mathfrak{M}}_{-1,0}$ by pulling back and $\mathfrak{M}_{-1,0}=\widetilde{\mathfrak{M}}_{-1,0} / C_{\hat{H}}$. Endow $\widetilde{\mathfrak{M}}_{-1,0}$ with the $C^{\infty}$ topology and $\mathfrak{M}_{-1,0}$ with the quotient topology. Let $\widetilde{\mathfrak{M}}_{-1,0}^{0}$ be the connected component of $\widetilde{\mathfrak{M}}_{-1,0}$, containing $(\hat{J}, \hat{\theta})$. Define the Teichmuller-type space $\mathfrak{T}_{-1,0}$ to be $\widetilde{\mathfrak{M}}_{-1,0}^{0} / C_{\hat{H}}^{0}$.

Corollary B. The map $\iota: \mathfrak{T}_{-1,0} \rightarrow \mathfrak{S}_{0}^{t}$ given by $\iota[(\hat{S}, \hat{H}, J, \theta)]=[(\hat{S}, \hat{H}, J)]$ is well defined and a homeomorphism.

Thus we can endow $\mathfrak{T}_{-1,0}$ with the smooth manifold structure induced from $\mathfrak{S}_{0}^{t}$ through $\iota$. 
The universal Picard variety (or Jacobian variety) plays an important role for many problems in algebraic geometry. Thus our differentialgeometric proof of the structure and dimension of the unramified universal Picard variety $P_{i c}^{t}$ has its own interest and merits an independent emphasis:

Theorem C. $P_{i c}^{t}$ is a complex manifold of (complex) dimension $4 g-3$.

In section 2 we prove some basic results about spherical CR circle bundles arising from holomorphic line bundles. In section 3 we prove Theorem C. We give a representation of the tangent space of $P_{i c}^{t}$ in the "classical gauge" (see (3.32)), which maps onto the space of holomorphic (1,0)-forms through $\bar{\partial}$-operator with the kernel equal to the space of holomorphic quadratic differentials relative to the reference Riemann surface. To parametrize our moduli space of spherical CR structures we introduce a certain local "supporting" manifold in section 4 . We also show the properness of the contact action in our case. In section 5 we parametrize $\mathfrak{S}_{0}^{t}$ as a smooth manifold with the aid of the map $\tau^{t}$ and the local "supporting" manifold. Finally we prove Theorem A and Corollary B. On the way to showing Theorem A, we actually obtain another representation of the tangent space of $P_{i c}^{t}$, which is a fourth-order differential equation. (It is basically because the deformation tensor of spherical CR structures in dimension 3 is of fourth order.) In Appendix A we prove the $U(1)$-invariant version of Gray's theorem (Theorem 5.1). In Appendix B we give a description of an infinitesimal slice of $\widetilde{\mathfrak{M}}_{-1,0} / C_{\hat{H}}$.

Our theory for the universal Picard variety has its counterpart in the Teichmuller theory as shown in the following table:

\begin{tabular}{c|c} 
Teichmuller space & universal Picard variety \\
\hline conformal classes & spherical CR circle bundles \\
\hline Riemannian hyperbolic metrics & pseudohermitian hyperbolic geometries
\end{tabular}

Our description of $P_{i c_{0}}^{t}$ using $\mathfrak{T}_{-1,0}$ (combining Theorem A and Corollary B) has a topological implication. Namely, the topology of (contact, hence) diffeomorphism group of $\hat{S}$ in principle can be determined by the topologies of $\mathfrak{T}_{-1,0}$ and the unimodulo representative space $\widetilde{\mathfrak{M}}_{-1,0}^{0}$. But the topology of $\mathfrak{T}_{-1,0}$ is the same as that of $P_{i c_{0}}^{t}$ by our theorems, which is well known. To study the topology of $\widetilde{\mathfrak{M}}_{-1,0}^{0}$, we might define a certain kind of Dirichlet's energy on it and use this energy functional as our Morse function. A similar strategy works successfully in studying the topology of Teichmuller space.([Tr]) 
Another problem is the analogue of the so-called Nielsen realization problem about the mapping class group of a Riemann surface. The Nielsen realization problem says whether any finite subgroup of the mapping class group Diff $f_{+} / D i f f_{0}$ (of a surface with genus $>1$ ) can be "realized" as a subgroup of $D i f f_{+}$. There is an analytic proof using the above- mentioned Dirichlet's energy and the so-called Weil-Petersson metric.([Tr]) We wonder if we can do the similar thing for a 3-manifold of circle bundle type through the study of $\mathfrak{T}_{-1,0}$.

As we know, the moduli space of Riemann surfaces of fixed genus is the quotient of Teichmuller space by the mapping class group. The compactification of the moduli space has been well studied. There are a couple of ways to do it. The way using algebraic geometry was first done by Deligne and Mumford. It was realized later a different approach which is based on the Riemannian hyperbolic geometry. (e.g., [SS], [Pa]) As for the compactification of the universal Picard variety, algebraic approaches have been taken up by several authors. ([Ds], [Is], [OS], [Cap], etc.) Towards the problem of compactification we hope that along with the framework of this paper there will be a differential-geometric approach in the near future.

\section{Spherical CR circle bundles.}

Let $L$ be a negative holomorphic line bundle over a closed Riemann surface $N$ of genus $g>1$. For such $N$, there always exists a unique hyperbolic metric $d s^{2}$ (i.e. the associated Gaussian curvature $K_{d s^{2}}=-1$ ) in its associated conformal class. Denote the volume form of $d s^{2}$ by $\omega_{d s^{2}}$. By the GaussBonnet theorem the integral of $\hat{\omega}_{d s^{2}}=-\omega_{d s^{2}} / 2 \pi \chi(N)=\omega_{d s^{2}} / 4 \pi(g-1)$ equals 1. Hence $\left[\hat{\omega}_{d s^{2}}\right]$ is a generator of $H^{2}(N, Z)=Z$. Write $c_{1}(L)=$ $-m\left[\hat{\omega}_{d s^{2}}\right]$ for $m$ being a positive integer.

Proposition 2.1. There exists a unique (up to a positive constant multiple) hermitian metric \|\|$: L \rightarrow \mathbb{R}^{+} \cup\{0\}$ such that if we write $h(z, \bar{z})=\|s(z)\|^{2}$ for a local holomorphic section s, then

$$
i \partial_{z} \partial_{\bar{z}} \log h(z, \bar{z})=(m /(2 g-2)) \omega_{d s^{2}}
$$

Proof. Take an arbitrary hermitian metric \|\|$_{0}$ and write $h_{0}(z, \bar{z})=\|s(z)\|_{0}^{2}$. Any other hermitian metric \|\|$^{2}$ is equal to $\lambda\|\|_{0}^{2}$ for $\lambda$ being a global positive function defined on $N$. It suffices to solve $\lambda$ for the following equation:

$$
i \partial_{z} \partial_{\bar{z}} \log \lambda(z, \bar{z})=(m /(2 g-2)) \omega_{d s^{2}}-i \partial_{z} \partial_{\bar{z}} \log h_{0}(z, \bar{z})
$$


Equating and then multiplying coefficients of $i d z \wedge d \bar{z}$ in (2.2) by $g^{1 \overline{1}}=$ $\left(g_{1 \overline{1}}\right)^{-1}$ where $d s^{2}=g_{1 \overline{1}} d z d \bar{z}$ gives

$$
\frac{1}{2} \Delta_{d s^{2}} \log \lambda=\Sigma
$$

where $\Sigma$ is a global real function. Note that both $(m /(2 g-2)) \omega_{d s^{2}}$ and $i \partial_{z} \partial_{\bar{z}} \log h_{0}(z, \bar{z})$ represent $-2 \pi c_{1}(L)$. It follows that $\int \Sigma \omega_{d s^{2}}=0$. So we can solve (2.3) for $\lambda$ unique up to a positive constant multiple (see, for instance, p.104 in $[\mathrm{Au}])$ and hence (2.2).

Define the circle bundle $S_{L} \subset L$ by \|\|$=1$. The contact bundle and the CR structure on $S_{L}$, induced from L, are denoted $H_{L}, J_{L}$ respectively. Define the contact form $\theta_{L}$ on $S_{L}$ by

$$
\theta_{L}=-\left.i \kappa \partial_{L}\left(\|\|^{2}\right)\right|_{S_{L}}
$$

with the normalizing constant $\kappa=2(g-1) / m$. Locally write $\|w s(z)\|^{2}=$ $h(z, \bar{z})|w|^{2}$ for $w$ in $\mathbb{C}$, a fibre coordinate. A direct computation using (2.1) shows that

$$
d \theta_{L}=\pi^{*} \omega_{d s^{2}} \quad\left(\pi: S_{L} \subset L \rightarrow N \text { being the bundle projection }\right)
$$

Let $w^{1}, w^{2}$ be orthonormal coframe fields relative to $d s^{2}$. Let $\theta^{1}=w^{1}+$ $i w^{2}, \theta^{\overline{1}}=w^{1}-i w^{2}$ be the corresponding unitary coframe fields. Hence $\omega_{d s^{2}}=w^{1} \wedge w^{2}=(1 / 2) i \theta^{1} \wedge \theta^{\overline{1}}$. From the formulas on pp. 266-267 in [We2], the pseudohermitian scalar (or Tanaka-Webster) curvature

$$
\begin{aligned}
R_{J_{L}, \theta_{L}} & =H^{1 \overline{1}} R_{1}{ }^{1}{ }_{1 \overline{1}} \quad\left(\text { since } h_{1 \overline{1}}=\frac{1}{2}, \quad R_{1}{ }^{1}{ }_{1 \overline{1}}=(1 / 2) K_{d s^{2}}\right) \\
& =2(1 / 2) K_{d s^{2}}=K_{d s^{2}}=-1
\end{aligned}
$$

and the torsion

$$
A_{J_{L}, \theta_{L}}=0
$$

Therefore by (2.4) in [CL1] the Cartan (curvature) tensor

$$
Q_{J_{L}}=0
$$

It follows ([Ca], $[\mathrm{CM}])$ that $\left(S_{L}, H_{L}, J_{L}\right)$ is spherical, i.e., locally CRequivalent to the unit sphere $S^{3}$ in $\mathbb{C}^{2}$. The following Proposition shows the uniqueness of the contact form $\theta_{L}$ in (2.5). 
Proposition 2.2. Let $(M, H)$ be a closed contact manifold of dimension 2n+1. Let $\left(J, \theta_{j}\right), j=1,2$ be pseudohermitian structures on $(M, H)$. (i.e. $J$ is compatible with $H$ and $\theta_{j}$ 's are contact forms relative to $H$ ) Suppose the pseudohermitian scalar curvature $R_{J, \theta_{j}}=-1, j=1,2$. Then $\theta_{1}=\theta_{2}$.

Proof. Write $\theta_{2}=u^{2 / n} \cdot \theta_{1}$ for $u>0 . R_{J, \theta_{1}}$ and $R_{J, \theta_{2}}$ are related in the following equation:

$$
(2(n+1) / n) \Delta_{b} u+R_{J, \theta_{1}} u-R_{J, \theta_{2}} u^{(n+2) / n}=0
$$

(see [JL]; note that $\Delta_{b}$ is the "negative" sublaplacian relative to $\left(J, \theta_{1}\right)$ ) Substituting $R_{J, \theta_{j}}=-1$ in (2.8) gives

$$
\frac{2(n+1)}{n} \Delta_{b} u=u-u^{(n+2) / n}
$$

Suppose $\mathrm{u}$ achieves its maximal value $>1$ at a point $p$. Then we evaluate (2.9) at $p:$

$$
0 \leq \frac{2(n+1)}{n} \Delta_{b} u=u-u^{\frac{n+2}{n}}<0
$$

to reach a contradiction. Similarly u cannot achieve its minimal value $<1$. Therefore $\mathrm{u}$ must be identically equal to 1 .

Corollary 2.3. The map $\iota$ in Corollary $B$ (assuming it is well defined) is injective.

Next suppose we have a holomorphic bundle isomorphism $(\phi, f)$ : $\left(L_{1}, N_{1}\right) \rightarrow\left(L_{2}, N_{2}\right)$ for $\left[\left(L_{j}, N_{j}\right)\right] \in P_{i c}, j=1,2$. By Proposition 2.1 and noting that the biholomorphism $f: N_{1} \rightarrow N_{2}$ is an isometry, we conclude that

$$
\phi^{*}\left(\|\|_{L_{2}}\right)=c\|\|_{L_{1}}
$$

for some constant $c>0$, where \|\|$_{L_{j}}, j=1,2$ denote hermitian metrics obtained in Proposition 2.1 with respect to $L_{j}$. Let $m_{c}$ denote the multiplication by $c$ on the line bundle. Thus by (2.10) the composition $\phi \circ m_{c}^{-1}: S_{L_{1}} \rightarrow S_{L_{2}}$ is a CR equivalence. We have shown that the map $\tau:[(L, N)] \rightarrow\left[\left(S_{L}, H_{L}, J_{L}\right)\right]$ is well-defined. Furthermore, we have

Proposition 2.4. The map $\tau$ is injective. 
Proof. Let $D_{L}$ denote the disc bundle of $L$ with the boundary $\partial D_{L}=S_{L}$. Since $S_{L}$ is the strictly pseudoconvex boundary of the complex manifold $D_{L}$, it is CR-embeddable in $\mathbb{C}^{N}$ and coordinate functions (CR functions on $S_{L}$ ) extend holomorphically to $D_{L}$. (see, e.g., Theorem 5.3 in [K1], Corollary of Theorem 1.3 in [K2], p.91(5.3.5) in [FK]) So we have a map $\psi: \bar{D}_{L} \rightarrow \mathbb{C}^{N}$, holomorphic in $D_{L}$, and CR equivalent between $\partial \bar{D}_{L}=S_{L}$ and $\psi\left(S_{L}\right)$. Denote the 0 -section of $D_{L}$ by $\Sigma$. $\Sigma$ is biholomorphic to the closed Riemann surface $N$. We claim $\psi: D_{L} \backslash \Sigma \rightarrow \mathbb{C}^{N}$ is a biholomorphism onto its image. First observe that $\psi$ is biholomorphic near the boundary $S_{L}$ and the disc bundle $D_{L}(\rho)=\left\{s \in D_{L}:\|s\|_{L}<\rho\right\}$ of radius $\rho, 0<\rho<1$, is strictly pseudoconvex. By continuity, there exists a smallest $\rho_{0} \geq 0$ such that $\psi$ is biholomorphic on $D_{L} \backslash D_{L}\left(\rho_{0}\right)$ and fails to be biholomorphic on $S_{L}\left(\rho_{0}\right)=\partial \overline{D_{L}\left(\rho_{0}\right)}$. Suppose $\rho_{0}>0$. Take $q \in S_{L}\left(\rho_{0}\right)$. Near q consider the determinant of the Jacobian matrix of $\psi$, denoted $J_{\psi}$. If $J_{\psi}(q)=0$, the subvariety defined by $J_{\psi}=0$ must contain a point near $q$ but out of $\overline{D_{L}\left(\rho_{0}\right)}$ due to pseudoconvexity of $D_{L}\left(\rho_{0}\right)$, which contradicts $\psi$ being biholomorphic on $D_{L} \backslash \overline{D_{L}\left(\rho_{0}\right)}$ (where $J_{\psi} \neq 0$ ). Thus $J_{\psi}(q) \neq 0$. Hence $\psi$ is a local biholomorphism near $S_{L}\left(\rho_{0}\right)$. Therefore $\psi$ must be globally injective near $S_{L}\left(\rho_{0}\right)$ since it is biholomorphic on "one side" of $S_{L}\left(\rho_{0}\right)$. In conclusion $\rho_{0}$ must be 0 and we have proved our claim.

Now take two holomorphic line bundles $\left(L_{j}, N_{j}\right), j=1,2$ with associated spherical CR circle bundles $S_{L_{j}}$ 's being isomorphic. That is to say, there exists a CR equivalence $\phi: S_{L_{1}} \rightarrow S_{L_{2}}$. As just discussed above, there exists a map $\psi_{1}: \bar{D}_{L_{1}} \rightarrow \mathbb{C}^{N}$, biholomorphic on $D_{L_{1}} \backslash \Sigma_{1}\left(\Sigma_{j}\right.$ 's, $j=1,2$, denote zero sections of $L_{j}$ respectively) Moreover the CR embedding $\psi_{1} \circ \phi^{-1}$ : $S_{L_{2}} \rightarrow \mathbb{C}^{N}$ extends to a map $\psi_{2}: \bar{D}_{L_{2}} \rightarrow \mathbb{C}^{N}$, biholomorphic on $D_{L_{2}} \backslash \Sigma_{2}$, with Range $\psi_{2}=$ Range $\psi_{1}$ by the uniqueness of solution for the complex Plateau problem in $\mathbb{C}^{N}$. ([HL], [Y]) Since $\Sigma_{j}$ 's are biholomorphic to closed Riemann surfaces $N_{j}$ 's respectively, $\psi_{j}\left(\Sigma_{j}\right)$ consists of a point $p_{j}$ in $\mathbb{C}^{N}$. Suppose $p_{1} \neq p_{2}$. Take a suitable neighborhood $U$ of $p_{2}$ such that $\psi_{1}^{-1}\left(U \backslash p_{2}\right)$ is biholomorphic to $\psi_{2}^{-1}\left(U \backslash p_{2}\right)$. But they have different topological types since the latter is a tubular neighborhood of a closed Riemann surface $\Sigma_{2}$. So $p_{1}=p_{2}$ and $\phi_{1}=\psi_{2}^{-1} \circ \psi_{1}: D_{L_{1}} \backslash \Sigma_{1} \rightarrow D_{L_{2}} \backslash \Sigma_{2}$ is a biholomorphism. Furthermore it is easy to see that a punctured fibre disc must be mapped by $\phi_{1}$ onto a punctured disc with the puncture sitting in $\Sigma_{2}$. (just noting that the puncture in $\Sigma_{1}$ is a removable singularity) We therefore extend $\phi_{1}$ to a map (still denoted $\phi_{1}$ ) from $D_{L_{1}}$ into $D_{L_{2}}$ carrying $\Sigma_{1}$ into $\Sigma_{2}$. We claim $\phi_{1}$ is continuous on $\Sigma_{1}$. Take $q \in \Sigma_{1}$ and $\widetilde{q}=\phi_{1}(q) \in \Sigma_{2}$. Centered at $q$, we have local holomorphic coordinates $(z, w) \in D \times D^{*}$ for $\Sigma_{1} \times$ fibres where 
$D$ ( $D^{*}$ resp.) denotes the (punctured resp.) disc. Given neighborhoods $U$ and $V$ of $\widetilde{q}$ in $D_{L_{2}}$ with $\bar{V} \subset U$, there exists a positive number $r$ such that $\{(0, w):|w|<r\}$ is mapped into $V$. Observe that

$$
d_{D_{L_{2}} \backslash \Sigma_{2}}\left(\phi_{1}(z, w), \phi_{1}(0, w)\right) \leq d_{D \times D^{*}}((z, w),(0, w)) \leq d_{D}(z, 0)
$$

where " $d$ " denotes the Kobayashi distance. Let

$$
b=d_{D_{L_{2}} \backslash \Sigma_{2}}\left(\left(D_{L_{2}} \backslash \Sigma_{2}\right) \cap V,\left(D_{L_{2}} \backslash \Sigma_{2}\right) \backslash U\right)>0 .
$$

Then there is a positive number $r^{\prime}$ such that $d_{D}(z, 0)<b$ for $|z|<r^{\prime}$, so it follows by (2.11) that $\phi_{1}(z, w)$ is in $U$ for $|z|<r^{\prime},|w|<r$. Once we know $\phi_{1}$ is continuous on $\Sigma_{1}$, then it must be holomorphic on $D_{L_{1}}$ by the Riemann extension theorem. (we can also just invoke Theorem 6.2 in [Ko] p.93 to replace the above argument) Similarly extend $\phi_{1}^{-1}$ holomorphically to $D_{L_{2}}$. Since the holomorphic map $\phi_{1}^{-1} \circ \phi_{1}=$ identity on $D_{L_{1}} \backslash \Sigma_{1}$, it must be an identity on $D_{L_{1}}$. We have shown that $\phi_{1}$ is a biholomorphism between $D_{L_{1}}$ and $D_{L_{2}}$. Define biholomorphisms $\phi_{\rho}: D_{L_{1}}(\rho) \rightarrow D_{L_{2}}(\rho)$ for $\rho>0$ by $\phi_{\rho}(y)=\rho \phi_{1}(y / \rho)$. In local coordinates $(z, w)$ with $\mathrm{w}$ being fibre coordinate, we can write $\phi_{\rho}=(\widetilde{z}, \widetilde{w})$ as a function of $y=(z, w)$ : at $z=0$,

$$
\widetilde{z}=O(w / \rho), \widetilde{w}=c w+O\left(w^{2} / \rho\right)
$$

for some nonzero constant $c$. As $\rho$ goes to infinity, $\widetilde{z}$ approaches to 0 and $\widetilde{w}$ goes to $c w$. That is to say, $\lim _{\rho \rightarrow \infty} \phi_{\rho}=\phi_{\infty}: L_{1} \rightarrow L_{2}$ exists and is a linear isomorphism on each fibre, and from the above argument $\left\{\phi_{\rho}=(\widetilde{z}, \widetilde{w})\right\}$ is uniformly bounded on any compact coordinate neighborhood around a point. It follows that $\phi_{\infty}$ is holomorphic. Apply a similar argument to $\phi_{\rho}^{-1}(x)=\rho \phi_{1}^{-1}(x / \rho)$. We obtain a holomorphic map $\psi=\lim \phi_{\rho}^{-1}: L_{2} \rightarrow L_{1}$ and it is easy to see that $\psi \circ \phi_{\infty}=\phi_{\infty} \circ \psi=$ identity. Therefore $\phi_{\infty}$ is a holomorphic bundle isomorphism.

\section{Parametrizing $P_{i c}^{t}$ as complex manifold: Proof of Theorem C.}

First we describe $P_{i c}$ by complex structures with special properties on the fixed background line bundle $\hat{L}$ (considered as a smooth line bundle). Since every holomorphic line bundle $(L, N)$ of the fixed $c_{1}$ is isomorphic to $(\hat{L}, \hat{N})$ as smooth line bundle, complex structures on $L$ and $N$ are pulled back to $\hat{L}$ and $\hat{N}$ respectively. Let Bdiff denote the group of smooth bundle automorphisms of $(\hat{L}, \hat{N})$. Let $J, c$ denote complex structures on $\hat{L}, \hat{N}$ respectively. The space of all $((\hat{L}, J),(\hat{N}, c))$ such that the projection from $\hat{L}$ 
onto $\hat{N}$ is holomorphic with respect to $(J, c)$ modulo Bdiff is in one-to-one correspondence with $P_{i c}$. Let $m_{\rho}: \hat{L} \rightarrow \hat{L}$ denote the fibre multiplication by $\rho$, a complex number. Let $\mathbb{C}^{*}$ denote the subgroup of $B \operatorname{diff}$, consisting of all $m_{\rho}$ with nonzero $\rho$. Let $\hat{J}$ denote the complex structure on $\hat{L}$ (and also on $\hat{S}$, cf. section 1) associated to the fixed (or reference) holomorphic line bundle $(\hat{L}, \hat{N})$. On $\hat{L}$ we consider the space $\widetilde{P}_{i c}$ of all smooth almost complex structures $J$ respecting the same orientation as given by $\hat{J}$ on $\hat{L}$ and satisfying the following conditions:

(3.1) $m_{\rho}^{*} J=J$ for nonzero $\rho$ (i.e. $J$ is $\mathbb{C}^{*}$-invariant) and

(3.2) on fibres, $J$ is induced by the usual complex structure on $\mathbb{C}$ in local trivializations.

Proposition 3.1. Any $J$ in $\widetilde{P}_{i c}$ is integrable.

Proof. First fix a system of local coordinates $(z, w)$ on $\hat{L}$ (holomorphic with respect to the original reference complex structure $\hat{J}$ on $\hat{L}$ ) with fibre coordinate $w$. Let $\partial_{z}, \partial_{w}$ denote the tangent vectors $\partial / \partial z, \partial / \partial w$ respectively for short. The condition (3.1) allows us to construct a $\mathbb{C}^{*}$-invariant $(1,0)$ (relative to $J$ ) tangent vector $Z_{1}$ on $\hat{L}$ by moving a chosen $(1,0)$ section by the action of $\mathbb{C}^{*}$. Write

$$
Z_{1}=f \partial_{z}+g \partial_{\bar{z}}+h w \partial_{w}+\ell \bar{w} \partial_{\bar{w}}
$$

$\mathbb{C}^{*}$-invariance implies that $f, g, h, l$ are smooth functions only in $z, \bar{z}$. It follows that $\left[Z_{1}, \partial_{w}\right]=-h \partial_{w}$. Now we can compute the Nijenhuis tensor:

$$
N\left(Z_{1}, \partial_{w}\right)=-4\left[Z_{1}, \partial_{w}\right]-4 i J\left[Z_{1}, \partial_{w}\right]=0,
$$

and it is easy to see that

$$
N\left(\partial_{w}, \partial_{w}\right)=N\left(\partial_{w}, \partial_{\bar{w}}\right)=N\left(Z_{1}, \partial_{\bar{w}}\right)=N\left(Z_{1}, Z_{\overline{1}}\right)=0 .
$$

Thus by noting that $N$ is skew-symmetric, the Nijenhuis tensor vanishes.

Observe that $(\hat{L}, J)$ in $\widetilde{P}_{i c}$ can be pushed down to $(\hat{N}, c)$ for some complex structure $c$. (for $v$ tangent to $\hat{N}, c(v)$ is defined to be $\hat{\pi}_{*} J\left(s_{0 *}(v)\right.$ ). Here $s_{0}$ is the zero section of $\hat{L}$ over $\hat{N}$ and $\hat{\pi}: \hat{L} \rightarrow \hat{N}$ is the natural projection. It follows that $\hat{\pi}$ is holomorphic with respect to $(J, c))$. Hence the quotient space $\widetilde{P}_{i c} / B$ diff is in one-to-one correspondence with $P_{i c}$. Let 
$B$ dif $f_{0}$ denote the group of smooth bundle automorphisms $(\widetilde{\phi}, \phi)$ of $(\hat{L}, \hat{N})$ with $\phi: \hat{N} \rightarrow \hat{N}$ being isotopic to the identity. Denote the quotient group $B$ dif $f_{0} / \mathbb{C}^{*}$ by $\mathfrak{B}$. (note that $\mathbb{C}^{*}$ is contained in the center of $B$ dif $f_{0}$ ) Define the Teichmuller-type space $P_{i c}^{t}$ to be $\widetilde{P}_{i c} / \mathfrak{B}=\widetilde{P}_{i c} / B$ diff $f_{0}$. We are going to show that $\mathfrak{B}$ acts freely and properly on $\widetilde{P}_{i c}$ and $P_{i c}^{t}$ can be parametrized as complex manifold. First parametrize $\widetilde{P}_{i c}$ and $\mathfrak{B}$. Let us do a priori computation of the tangent space of $\widetilde{P}_{i c}$ at a reference point $\hat{J}$. Denote $J_{t}$ a family of elements in $\widetilde{P}_{i c}$ with $J_{0}=\hat{J}$. Let $E$ be the derivative of $J_{t}$ in $t$ at $t=0$ (considered in the space of endomorphisms of $T \hat{L}$ ). $J_{t}$ being almost complex structures implies that $E$ satisfies the following equation:

$$
E \circ \hat{J}+\hat{J} \circ E=0
$$

Take local holomorphic coordinates $(z, w)$ relative to $\hat{J}$ as in the proof of Proposition 3.1. Write $\partial_{b}=\partial / \partial z^{b}, b=1,2$ for short where $z^{1}=z, z^{2}=w$. We express $E$ as below:

$$
E=\Sigma E_{a}^{b} d z^{a} \otimes \partial_{b}+E_{a}^{\bar{b}} d z^{a} \otimes \partial_{\bar{b}}+\text { conjugate. }
$$

It follows from (3.3) that

$$
E_{a}{ }^{b}=0 \quad\left(\text { hence } E_{\bar{a}}^{\bar{b}}=\overline{\left(E_{a}^{b}\right)}=0\right)
$$

Condition (3.2) means $J_{t}\left(\partial_{2}\right)=i \partial_{2}$ whose differentiation in $t$ at $t=0$ gives $E\left(\partial_{2}\right.$ or $\left.\partial_{\overline{2}}\right)=0$. Hence we have

$$
E_{2}{ }^{\overline{1}}=E_{2}{ }^{\overline{2}}=0
$$

Besides, differentiating (3.1) tells that $E$ is $\mathbb{C}^{*}$-invariant. Therefore both $E_{1}{ }^{\overline{1}}$ and $E_{1}{ }^{\overline{2}} / \bar{w}$ are independent of the variable $w=z^{2}$. Together with (3.4),(3.5) we obtain

$$
E=E_{1}{ }^{\overline{1}}(z, \bar{z}) d z \otimes \partial_{\bar{z}}+E_{1}{ }^{*}(z, \bar{z}) \bar{w} d z \otimes \partial_{\bar{w}}+\text { conjugate }
$$

where $E_{1}{ }^{*}(z, \bar{z})$ is just $E_{1}{ }^{\overline{2}} / \bar{w}$. $E_{1}{ }^{\overline{1}}$ and $E_{1}{ }^{*}$ satisfy the transformation law:

$$
\begin{gathered}
\widetilde{E}_{1}{ }^{\overline{1}}=E_{1}{ }^{\overline{1}} \overline{\left(h^{\prime}\right)}\left(h^{\prime}\right)^{-1} \\
\widetilde{E}_{1}{ }^{*}=E_{1}{ }^{*}\left(h^{\prime}\right)^{-1}+E_{1}{ }^{\overline{1}}\left(h^{\prime}\right)^{-1} \overline{g^{\prime} g^{-1}}
\end{gathered}
$$

under the coordinate change of trivializations:

$$
\widetilde{z}=h(z), \widetilde{w}=g(z) w
$$


for biholomorphic $h$ and nonzero holomorphic $g$. Therefore we can talk about smooth or $H^{s}$ (Sobolev $s$-norm bounded) $E$ if $E_{1}{ }^{\overline{1}}$ and $E_{1}{ }^{*}$ are smooth or $H^{s}$. (the Sobolev $s$-norm can be defined via either a chosen partition of unity or a chosen covariant derivative on $\hat{N}$ ) Similarly by conditions (3.1), (3.2), we can write an element $J$ in $\widetilde{P}_{i c}$ as

$$
J=\Sigma J_{1}^{b} d z \otimes \partial_{b}+i d w \otimes \partial_{w}+\text { conjugate }
$$

where b runs over $1, \overline{1}, 2, \overline{2}$ and $J_{1}{ }^{1}, J_{1}{ }^{1}, J_{1}{ }^{2} / w, J_{1}{ }^{2} / \bar{w}$ are independent of $w$. Therefore we can talk about $H^{s} J$ if these components are all in $H^{s}$. Let $\widetilde{P}_{i c_{s}}$ denote the set of all such $H^{s} J$. Let $\mathfrak{E}_{\hat{J}}\left(\mathfrak{E}_{\hat{J}}^{s}\right.$ resp.) denote the linear space of all smooth $\left(H^{s}\right.$, resp.) tensors $E$ of the type (3.6). Since $\hat{N}$ is compact, $\mathfrak{E}_{\hat{J}}$ is a tame Frechet space in the terminology of $[\mathrm{H}]$ while $\mathfrak{E}_{\hat{J}}^{s}$ is a Hilbert (hence Banach) space. Define a map $\Phi_{\hat{J}}: \mathfrak{E}_{\hat{J}} \rightarrow \widetilde{P}_{i c}$ by

$$
\Phi_{\hat{J}}(E)=(I-(1 / 2) E \circ \hat{J}) \circ \hat{J} \circ(I-(1 / 2) E \circ \hat{J})^{-1}
$$

for small (in $C^{\infty}$-topology) $E$. It is easy to see that $\Phi_{\hat{J}}$ extends to $\mathfrak{E}_{\hat{J}}^{s}$ (still denoted $\Phi_{\hat{J}}$ ) with the range $\widetilde{P}_{i c_{s}}$ for large enough s, say, $s \geq 2$ by the Sobolev lemma. (for $s \geq 2, H^{s}$-space is contained in $C^{0}$ and forms an algebra. Note also that the inverse of a nonzero $H^{s}$ function on $\hat{N}$ is still in $H^{s}$ ) Moreover $\Phi_{\hat{J}}$ is injective for small $E$ in $\mathfrak{E}_{\hat{J}}^{s}$ as the inverse $\Phi_{\hat{J}}^{-1}$ can be given precisely by

$$
\Phi_{\hat{J}}^{-1}(J)=2(J-\hat{J})(J+\hat{J})^{-1} \hat{J},
$$

and it is easy to compute that $(d / d t) \Phi_{\hat{J}}(t E)=E$ at $t=0$. (consider $\widetilde{P}_{i c}$ sitting in $\operatorname{End}(T \hat{L}))$ We use $\left\{\Phi_{J}: J \in \widetilde{P}_{i c}\right.$ or $\left.\widetilde{P}_{i c_{s}}\right\}$ to parametrize $\widetilde{P}_{i c}$ or $\widetilde{P}_{i c_{s}}$. The transition map for the overlap region have the precise formula by composing (3.10) and (3.9) for two different $\hat{J}$ 's. Observe that, with respect to a basis, each component of the transition map is a polynomial in components of $E$. It follows that the transition map is $C^{\infty}$ (smooth tame in the smooth case) and hence a $C^{\infty}$-diffeomorphism by symmetry. We have proved

Proposition 3.2. $\widetilde{P}_{i c_{s}}\left(\widetilde{P}_{i c}\right.$, resp.) is a smooth Hilbert (tame Frechet, resp.) manifold for $s \geq 2$.

Next a priori computation shows that a tangent vector of $B$ dif $f_{0}$ at the identity has the following form:

$$
X=v^{1} \partial_{z}+v^{\overline{1}} \partial_{\bar{z}}+v^{*} w \partial_{w}+\bar{v}^{*} \bar{w} \partial_{\bar{w}}
$$


in a local trivialization $(z, w)$ as above, where $v^{1}$ and $v^{*}$ are independent of $w$ and satisfy the following transformation law:

$$
\left\{\begin{array}{l}
\widetilde{v}^{1}=v^{1} h^{\prime}(z) \\
\widetilde{v}^{*}=v^{*}+v^{1} g^{\prime}(z) g(z)^{-1}
\end{array}\right.
$$

for the change of trivializations (3.8). Let $\tilde{\mathfrak{V}}^{s}$ denote the Hilbert space of all $X$ satisfying (3.12) with bounded $H^{s}$-norm. (may be defined by fixing a finite number of trivializations and a corresponding partition of unity for $\hat{N}$ so that the $H^{s}$-norm is locally provided by the sum of $H^{s}$-norms of $v^{1}$ and $v^{*}$ ) On the other hand a bundle automorphism $\phi$ of $\hat{L}$ can be expressed as

$$
\phi:(z, w) \rightarrow(\psi(z, \bar{z}), \lambda(z, \bar{z}) w)
$$

in trivialization $(z, w)$, where $\psi, \lambda$ obey the following transformation law:

$$
\begin{aligned}
& \widetilde{\psi}(h(z), \overline{h(z)})=h(\psi(z, \bar{z})) \\
& \tilde{\lambda}(h(z), \overline{h(z)})=\lambda(z, \bar{z}) g(\psi(z, \bar{z})) g(z)^{-1}
\end{aligned}
$$

according to (3.8). We say $\phi$ is $H^{s}$ if $\psi$ and $\lambda$ are $H^{s}$ for each trivialization. Let $B$ diff $f_{0}^{s}$ denote the topological space of all $H^{s}$ bundle automorphisms of $\hat{L}$ with obvious $H^{s}$-topology. Take $X$ in $\widetilde{\mathfrak{V}}^{s}$. We want to associate a bundle automorphism $\phi_{X}$ in Bdiff $f_{0}^{s}$. Take a (smooth) metric $d s^{2}$ on $\hat{N}$ and a hermitian connection $\nabla$ of $\hat{L}$ over $\hat{N}$. Let $V=\hat{\pi}_{*} X$ be the projection of $X$ on $\hat{N}$. Locally $V=v^{1} \partial_{z}+v^{\overline{1}} \partial_{\bar{z}}$ if $X$ is expressed as in (3.11). Let $\gamma(p, V(p), t)$ be the geodesic relative to $d s^{2}$ with initial point $p$ and initial velocity $V(p)$. It is well known that $\gamma$ is smooth in $(p, v, t)$ where defined. Let $s_{0}$ denote the local section of $\hat{L}$ given by $(z, 1)$. Define the connection form $\Gamma$ on $\hat{N}$ by

$$
\nabla_{v}\left(s_{0}\right)=\Gamma(v) s_{0}
$$

for tangent vectors $v$ on $\hat{N}$. Denote $(d / d t) \gamma(p, V(p), t)$ by $\gamma_{t}^{\prime}(p, V(p), t)$. In trivialization $(z, w)$, we identify $p$ with $z_{0}$ and move the fibre element $w_{0}$ parallelly according to (3.13) along the geodesic $\gamma\left(z_{0}, V\left(z_{0}\right), t\right)$ (instead of $\gamma\left(\left(z_{0}, \bar{z}_{0}\right), V\left(z_{0}, \bar{z}_{0}\right), t\right)$ for short) to get $w_{1}$ at time $=1$ (for small $V$ ). It is then easy to compute

$$
w_{1}=w_{0} \exp \left[-\int_{0}^{1} \Gamma\left(\gamma_{t}^{\prime}\left(z_{0}, V\left(z_{0}\right), t\right)\right) d t\right]
$$


(3.14) suggests the following choice of $\phi_{X}$ :

$$
\phi_{X}\left(z_{0}, w_{0}\right)=\left(\gamma\left(z_{0}, V\left(z_{0}\right), 1\right), w_{1} \exp \left[v^{*}\left(z_{0}\right)+\Gamma\left(V\left(z_{0}\right)\right)\right]\right) .
$$

Here we write $v^{*}\left(z_{0}\right)$ instead of $v^{*}\left(z_{0}, \bar{z}_{0}\right)$ and recall $V=v^{1} \partial_{z}+v^{\overline{1}} \partial_{\bar{z}}$ and $v^{*}$ are local components of $X$ as expressed in (3.11). We claim the definition of $\phi_{X}$ given by (3.15) is independent of the choice of trivialization. Let $(\widetilde{z}, \widetilde{w})$ be another trivialization related to $(z, w)$ by (3.8). We have corresponding local section $\widetilde{s}_{0}$ given by $(\widetilde{z}, 1)$ and associated connection form $\widetilde{\Gamma}$. It is easy to see that $g(z) \widetilde{s}_{0}=s_{0}$ and

$$
\widetilde{\Gamma}=\Gamma-d g \cdot g^{-1} .
$$

Now applying (3.16) to $\gamma_{t}^{\prime}\left(z_{0}, V\left(z_{0}\right), t\right)$ gives $\widetilde{w}_{1}=g\left(z_{1}\right) w_{1}$ where $z_{1}=$ $\gamma\left(z_{0}, V\left(z_{0}\right), 1\right)$. (note that $\widetilde{w}_{0}=g\left(z_{0}\right) w_{0}$ and $\widetilde{w}_{1}$ is given according to (3.14)). From the transformation law (3.12) for $v^{*}$ we can easily show that $v^{*}\left(z_{0}\right)+\Gamma\left(V\left(z_{0}\right)\right)$ is invariant under the change of trivialization (3.8). Altogether we have proved our claim. Observe that $\Gamma$ is smooth and $\gamma$, hence $\gamma_{t}^{\prime}$ is also smooth in their arguments. It follows that $\phi_{X}$ is $H^{s}$ if $X$ is $H^{s}$. So we have defined a map $\Sigma: \widetilde{\mathfrak{V}}^{s} \rightarrow B d i f f_{0}^{s}$ by $\Sigma(X)=\phi_{X}$. If we write

$$
\phi_{X}(z, w)=\left(\phi_{X}^{1}(z, \bar{z}), w \exp \phi_{X}^{2}(z, \bar{z})\right)
$$

then $\phi_{X}^{1}$ gives rise to a global diffeomorphism on $\hat{N}$ (still denoted $\phi_{X}^{1}$ ) and the inverse of $\Sigma$ can be given by

$$
\begin{aligned}
V= & P^{-1}\left(\phi_{X}^{1}\right) \\
v^{*}= & \phi_{X}^{2}-\Gamma(V(\cdot))+\int_{0}^{1} \Gamma\left(\gamma_{t}^{\prime}(\cdot, V(\cdot), t)\right) d t \\
& (\text { with } V \text { replaced by the first formula) }
\end{aligned}
$$

Here $P$ is the usual map of parametrization from vector fields to diffeomorphisms on $\hat{N}$ via the geodesic flow. Now it is clear that $\Sigma$ is a homeomorphism from an open set of small $X$ to a neighborhood of the identity, say, $U$. Let $l_{\psi}$ denote the composition with $\psi$ from the left. By composing $\Sigma$ with $l_{\psi}$ for smooth elements $\psi$ in $\mathfrak{B}^{s}$, we obtain an atlas $\left\{\left(l_{\psi}(U), \Sigma^{-1} \circ l_{\psi^{-1}}\right): \psi\right.$ is a smooth element in $\left.B d i f f_{0}^{s}\right\}$ for $B d i f f_{0}^{s}$. (note that the set of smooth elements is dense in $B d i f f_{0}^{s}$, and the composition map and the map taking each diffeomorphism to its inverse are $C^{0}$ ) To show the transition map being smooth is a matter of direct verification (using (3.14); (3.15), (3.17)) : one only has to observe that composing with a smooth element is smooth in the 
original argument. (Actually we can prove $B \operatorname{dif} f_{0}^{s}$ is a topological group for $s \geq 3$ under the operation of composition of $H^{s}$-maps (cf. section 3 in [Eb]). However the composition map is only $C^{0}$ but not $C^{1}$, so to get $C^{\infty}$ differentiable structure on $B \operatorname{diff} f_{0}^{s}$, we have to restrict to smooth elements as our "centers" of charts) Let $\mathfrak{V}^{s}$ denote the quotient space $\widetilde{\mathfrak{V}}^{s} / C$ where $C$ consists of all $X$ in (3.11) with $v^{1}=0, v^{*}=a$ constant complex number.(this is well defined according to the transformation law (3.12)) Since any finite-dimensional subspace of a Hilbert space is closed, we can identify $\mathfrak{V}^{s}$ with the closed orthogonal complement of $C$ in $\widetilde{\mathfrak{V}}^{s}$, which inherits the Hilbert space structure from $\widetilde{\mathfrak{V}}^{s}$. Recall $\mathfrak{B}^{s}=B \operatorname{diff} f_{0}^{s} / \mathbb{C}^{*}$ where $\mathbb{C}^{*}$ consists of all fibre dilations by nonzero constant complex numbers. (see the beginning of this section) Observe that $C$ is mapped into $\mathbb{C}^{*}$ by $\Sigma$ through the exponential function according to (3.14), (3.15), so $\Sigma$ induces a homeomorphism from a neighborhood of 0 in $\mathfrak{V}^{s}$ to a neighborhood of the identity in $\mathfrak{B}^{s}$. Similar construction as for $B d i f f_{0}^{s}$ gives us the desired charts for $\mathfrak{B}^{s}$. We have proved

Proposition 3.3. Bdiff $f_{0}^{s}$ and $\mathfrak{B}^{s}$ are smooth Hilbert manifolds.

Next we consider the behavior of $\mathfrak{B}^{s+1}\left(\mathfrak{B}\right.$, resp.) acting on $\widetilde{P}_{i c_{s}}\left(\widetilde{P}_{i c}\right.$, resp.) by the pullback. (well-defined because $C^{*}$ is contained in the center of $B$ dif $f_{0}$ ) First we have

Proposition 3.4. $\mathfrak{B}^{s+1}$ acts freely on $\widetilde{P}_{i c_{s}}$ for $s \geq 4$; in particular, $\mathfrak{B}$ acts freely on $\widetilde{P}_{i c}$.

Proof. A bundle automorphism $\phi$ in $H^{s+1}$ fixing a complex structure in $\widetilde{P}_{i c_{s}}$ can be pushed down to an $H^{s+1}$-biholomorphism $\phi$ relative to the pushed down $H^{s}$-complex structure on $\hat{N}$. Since $\phi$ is isotopic to the identity map, it follows by a standard result for genus $\geq 2$ Riemann surfaces (e.g. p.39 in $[\operatorname{Tr}]$ ) that $\phi$ must itself be the identity map. (to apply the NewlanderNirenberg theorem [NN, Theorem 1.1], we require $s \geq 4$ so that $H^{s}$ is contained in $C^{2}$ ) Thus $\phi$ is just a fibre multiplication by a nonzero holomorphic function $\lambda$ on $\hat{N}$. Compactness of $\hat{N}$ implies $\lambda$ must be a constant $\rho$. Therefore $\phi=m_{\rho}$ belongs to $C^{*}$.

Proposition 3.5. $\mathfrak{B}^{s+1}$ acts properly on $\widetilde{P}_{i c_{s}}$ for $s \geq 4$ : i.e. if $\phi_{j}^{*} J_{j}=\widetilde{J}_{j}$ converges to $\widetilde{J}$ and $J_{j}$ converges to $J$ in $H^{s}$ with $J_{j}$ in $\widetilde{P}_{i c_{s}},\left[\phi_{j}\right]$ in $\mathfrak{B}^{s+1}$, then there exists a subsequence of $\left[\phi_{j}\right]$ which converges in $H^{s+1}$ to some $[\phi]$. 
Proof. First we have $\phi_{j}{ }^{*} J_{j}=\widetilde{J}_{j}$, so $\phi_{j}$ can be pushed down to a biholomorphism $\underline{\phi}_{j}$ from $\left(\hat{N}, \widetilde{c}_{j}\right)$ to $\left(\hat{N}, c_{j}\right)$, where $\widetilde{c}_{j}, c_{j}$ are pushed down complex structures of $\widetilde{J}_{j}, J_{j}$ respectively. There is a diffeomorphism between $H^{s}$ oriented complex structures and $H^{s}$ hyperbolic metrics of Gaussian curvature -1 on a closed surface of genus $\geq 2$ ([Tr]), so we can apply the Ebin-Palais theorem (Theorem 2.3.1 in [Tr]) to conclude that a subsequence of $\underline{\phi}_{j}$ converges in $H^{s+1}$ to some $\underline{\phi}$. Let $\underline{\phi}(p)=q$ for $p, q$ in $\hat{N}$. Take holomorphic coordinates $z, \widetilde{z}$ with respect to $\widetilde{c}=\lim \widetilde{c}_{j}, c=\lim c_{j}$ around p,q respectively so that $\underline{\phi}$ satisfies the $\bar{\partial}$-equation in these coordinates. Take local trivializations $(z, w)$ and $\left(z^{\prime}, w^{\prime}\right)$ of $\hat{L}$ (which may not be holomorphic with respect to $\widetilde{J}$ and $J$ respectively). Write $\phi_{j}$ in these local trivializations:

$$
\phi_{j}:(z, w) \rightarrow\left(z^{\prime}, w^{\prime}\right)=\left(\underline{\phi}_{j}(z, \bar{z}), u_{j}(z, \bar{z}) w\right)
$$

for large $j$. Here $\underline{\phi}_{j}$ tends to $\underline{\phi}$ in $H^{s+1}$ as $j$ goes to infinity. Moreover since $\underline{\phi}$ is holomorphic with respect to $z, \partial_{\bar{z}} \underline{\phi}_{j}$ goes to zero in $H^{s}$. (note that we need the $H^{s}$ version of the Newlander-Nirenberg theorem ([FK]) to conclude that $\underline{\phi}_{j}$ is still in $H^{s+1}$ with respect to the $z$-coordinate) Now write $J_{j}$ in $\left(z^{\prime}, w^{\prime}\right)$ :

$$
J_{j}=d z^{\prime} \otimes\left(f_{j} \partial_{z^{\prime}}+g_{j} \partial_{\bar{z}^{\prime}}+h_{j} w^{\prime} \partial_{w^{\prime}}+\ell_{j} \bar{w}^{\prime} \partial_{\bar{w}^{\prime}}\right)+i d w^{\prime} \otimes \partial_{w^{\prime}}+\text { conjugate }
$$

where $f_{j}, g_{j}, h_{j}, \ell_{j}$ are $H^{s}$ functions in $z, \bar{z}$ according to (3.1),(3.2). Moreover $J_{j}^{2}=-I$ implies that $f_{j}, g_{j}, h_{j}, \ell_{j}$ satisfy the following algebraic conditions:

$$
\begin{aligned}
& f_{j}^{2}+\left|g_{j}\right|^{2}=-1 \\
& g_{j}\left(f_{j}+\bar{f}_{j}\right)=0 \\
& h_{j}\left(f_{j}+i\right)+g_{j} \bar{\ell}_{j}=0 \\
& \ell_{j}\left(f_{j}-i\right)+g_{j} \bar{h}_{j}=0
\end{aligned}
$$

Similarly for $\widetilde{J}_{j}$ we write capital $F_{j}, G_{j}, H_{j}, L_{j}$ for corresponding coefficients in trivialization $(z, w)$. Computing $\phi_{j}^{*} J_{j}$ and comparing corresponding coefficients of $w d z \otimes \partial_{w}$ and $\bar{w} d z \otimes \partial_{\bar{w}}$ with $\widetilde{J}_{j}$, we obtain

$$
\begin{aligned}
& \left(i-f_{j}+e_{j}^{1}\right) \frac{\partial}{\partial z}\left(u_{j}\right)-\left[\underline{\phi}^{\prime}(z)\left(\overline{\phi^{\prime}(z)}\right)^{-1} g_{j}+e_{j}^{2}\right] \frac{\partial}{\partial \bar{z}}\left(u_{j}\right)=u_{j} \widetilde{H}_{j} \\
& \left(-i-f_{j}+e_{j}^{1}\right) \frac{\partial}{\partial z}\left(\bar{u}_{j}\right)-\left[\underline{\phi}^{\prime}(z)\left(\overline{\phi^{\prime}(z)}\right)^{-1} g_{j}+e_{j}^{2}\right] \frac{\partial}{\partial \bar{z}}\left(\bar{u}_{j}\right)=\bar{u}_{j} \widetilde{L}_{j}
\end{aligned}
$$


where $\widetilde{H}_{j}=H_{j}-\frac{\partial \underline{\phi}_{j}}{\partial z} h_{j}-\frac{\partial \bar{\phi}_{j}}{\partial z} \bar{\ell}_{j}, \widetilde{L}_{j}=L_{j}-\frac{\partial \underline{\phi}_{j}}{\partial z} \ell_{j}-\frac{\partial \bar{\phi}_{j}}{\partial z} \bar{h}_{j}$ and

$$
\begin{aligned}
& e_{j}^{1}=\frac{\partial z}{\partial \bar{z}^{\prime}} \frac{\partial \bar{z}^{\prime}}{\partial z}\left(f_{j}-\bar{f}_{j}\right)-\frac{\partial z}{\partial \bar{z}^{\prime}} \frac{\partial z^{\prime}}{\partial z} g_{j}-\frac{\partial z}{\partial z^{\prime}} \frac{\partial \bar{z}^{\prime}}{\partial z} \bar{g}_{j} \\
& e_{j}^{2}=\frac{\partial \bar{z}}{\partial z^{\prime}}\left(\frac{\partial z^{\prime}}{\partial z} f_{j}+\frac{\partial \bar{z}^{\prime}}{\partial z} \bar{g}_{j}\right)-\left[\underline{\phi}^{\prime}\left(\underline{\phi}^{\prime}\right)^{-1}-\frac{\partial z^{\prime}}{\partial z}\left(\overline{\frac{\partial z}{\partial z^{\prime}}}\right)\right] g_{j}+\left(\overline{\frac{\partial z}{\partial z^{\prime}}}\right) \frac{\partial \bar{z}^{\prime}}{\partial z} \bar{f}_{j}
\end{aligned}
$$

Here $\frac{\partial z^{\prime}}{\partial z}\left(\frac{\partial z}{\partial z^{\prime}}\right.$, etc., resp. $)$ means $\frac{\partial}{\partial z}\left(\underline{\phi}_{j}\right)\left(\frac{\partial}{\partial z^{\prime}}\left(\underline{\phi}_{j}\right)^{-1}\right.$, etc., resp. $)$. It is easy to see that $e_{j}^{1}$ and $e_{j}^{2}$ converge to zero in $H^{s}$ as $j$ goes to infinity since $\underline{\phi}_{j}$ goes to a biholomorphism $\underline{\phi}$, and obviously $\widetilde{H}_{j}$ and $\widetilde{L}_{j}$ converge to $H-\left(\frac{\partial \underline{\phi}}{\partial z}\right) h$ and $L-\left(\frac{\partial \underline{\phi}}{\partial z}\right) \ell$ in $H^{s}$ respectively, where $H=\lim H_{j}, h=$ $\lim h_{j}, L=\lim L_{j}, \ell=\lim \ell_{j}$. Let

$$
\begin{aligned}
& D_{j}=\left(i-f_{j}\right) \frac{\partial}{\partial z}-\underline{\phi}^{\prime}\left(\bar{\phi}^{\prime}\right)^{-1} g_{j} \frac{\partial}{\partial \bar{z}} \\
& D_{j}^{\prime}=\left(-i-f_{j}\right) \frac{\partial}{\partial z}-\underline{\phi}^{\prime}\left(\underline{\phi}^{\prime}\right)^{-1} g_{j} \frac{\partial}{\partial \bar{z}}
\end{aligned}
$$

and

$$
\begin{aligned}
D & =(i-f) \frac{\partial}{\partial z}-\underline{\phi}^{\prime}\left(\bar{\phi}^{\prime}\right)^{-1} g \frac{\partial}{\partial \bar{z}} \\
D^{\prime} & =(-i-f) \frac{\partial}{\partial z}-\underline{\phi}^{\prime}\left(\bar{\phi}^{\prime}\right)^{-1} g \frac{\partial}{\partial \bar{z}}
\end{aligned}
$$

where $f=\lim f_{j}, g=\lim g_{j}$. Taking the limit of (3.18b) gives $g=0$ or $R e f=0$. If $g=0$ at $p, f=i$ or $-i$ by the limiting form of (3.18a). Then either $D$ or $D^{\prime}$ is not zero at $p$ and equals $\pm 2 i \frac{\partial}{\partial z}$. Hence either $D$ or $D^{\prime}$ is elliptic in a neighborhood of $p$. On the other hand, if $g$ does not vanish at $p$, then $\operatorname{Re} f=0$ at $p$. Suppose $(i-f)(a-i b)-\phi^{\prime}\left(\bar{\phi}^{\prime}\right)^{-1} g(a+i b)=0$ at $p$ for real nonzero $a$ or $b$. Then it follows that the absolute value of $(i-f) / \underline{\phi}^{\prime}\left(\bar{\phi}^{\prime}\right)^{-1} g$ equals 1 . By (3.18a)(limiting version) and $\operatorname{Re} f=0$ at $p$, we get $f=i$, which implies $g=0$ by (3.18a) again, a contradiction. Therefore $a=b=0$. We have proved that $D$ is elliptic around $p$ in the case of $g(p)$ not equal to zero, so in any case we use either $D$ or $D^{\prime}$ to do our interior elliptic estimates for $u_{j}$. Now we write our equations (3.19) as follows:

$$
\begin{aligned}
\left(D_{j}+E_{j}\right) u_{j} & =u_{j} \widetilde{H}_{j} \\
\left(D_{j}^{\prime}+E_{j}\right) \bar{u}_{j} & =\bar{u}_{j} \widetilde{L}_{j} .
\end{aligned}
$$


Here the error operator $E_{j}=e_{j}^{1} \frac{\partial}{\partial z}+e_{j}^{2} \frac{\partial}{\partial \bar{z}}$. Let $a_{j}=u_{j}(p)$ and $\hat{u}_{j}=$ $\left(a_{j}\right)^{-1} u_{j}$. Then $\hat{u}_{j}$ satisfies the same equation (3.20) as $u_{j}$ does but with $\hat{u}_{j}(p)=1$. (note that $\left(m_{a_{j}^{-1}} \circ \phi_{j}\right)^{*} J_{j}=\widetilde{J}_{j}$. Let $U$ be a small disc centered at $p$, which is compactly contained in another small neighborhood $V$. Let $|\cdot|_{s, W}$ denote the $H^{s}$ norm on $W$. Let $D_{j}^{*}\left(D_{j}^{\prime *}\right.$, resp.) denote the formal adjoint operator of $D_{j}+E_{j}\left(D_{j}^{\prime}+E_{j}\right.$, resp.). It is easy to see that either $D_{j}^{*} \circ\left(D_{j}+E_{j}\right)$ or $D_{j}^{\prime *} \circ\left(D_{j}^{\prime}+E_{j}\right)$ is real positive self-adjoint, strictly and uniformly elliptic in a neighborhood $\tilde{V}$ of $p$ so that the constants $\gamma$ and $\nu$ in (9.47) of [GT] are independent of $j$ for large enough $j$. Choose small discs centered at $p, U, V_{j}, j=1, \ldots, s, V$ such that $U \subset V_{1} \subset V_{2} \subset \cdots \subset V_{s} \subset V \subset \widetilde{V}$ where each smaller disc is compactly contained in larger ones and $V$ is chosen so that we can apply Theorem 9.20 of [GT]. By standard interior elliptic estimates, we compute (in case $D=0$ at $p$, replace $D_{j}^{*} \circ\left(D_{j}+E_{j}\right)$ by $D_{j}^{* *} \circ\left(D_{j}^{\prime}+E_{j}\right)$ and $\hat{u}_{j}$ by $\left.\overline{\hat{u}_{j}}\right)$

$$
\begin{aligned}
\left|\hat{u}_{j}\right|_{s+1, U} & \lesssim\left|D_{j}^{*} \circ\left(D_{j}+E_{j}\right) \hat{u}_{j}\right|_{s-1, V_{1}}+\left|\hat{u}_{j}\right|_{0, V_{1}} \\
& \lesssim\left|D_{j}^{*}\left(\hat{u}_{j} \widetilde{H}_{j}\right)\right|_{s-1, V_{1}}+\left|\hat{u}_{j}\right|_{0, V_{1}} \quad(\text { by }(3.20 \mathrm{a})) \\
& \lesssim\left|\hat{u}_{j}\right|_{s, V_{1}} \quad(\text { by the interpolation inequality) } \\
& \lesssim\left|\hat{u}_{j}\right|_{s-1, V_{2}} \quad \text { (by the same argument as above) } \\
& \cdots \\
& \lesssim\left|\hat{u}_{j}\right|_{0, V}
\end{aligned}
$$

where $A \lesssim B$ means $A \leq k B$ for constant k independent of $\hat{u}_{j}$. On the other hand applying the Harnack estimates (Theorems 9.20, 9.22 in [GT]) to the equation:

$$
\left[D_{j}^{*} \circ\left(D_{j}+E_{j}\right)\right] \log \left|\hat{u}_{j}\right|=\operatorname{Re}\left(D_{j}^{*} \widetilde{H}_{j}\right)
$$

(noting that we apply theorems to $\log \left|\hat{u}_{j}\right|-\widetilde{\inf } \log \left|\hat{u}_{j}\right| \geq 0$ ) we obtain the estimate of the supremum norm on $V$ :

$$
\left|\hat{u}_{j}\right|_{L^{\infty}, V} \leq C
$$

where $C$ is a constant independent of large enough j. Combining (3.21) and (3.22) we get

$$
\left|\hat{u}_{j}\right|_{s+1, U} \leq C_{s}
$$

so there exists a subsequence (still denoted $\left\{\hat{u}_{j}\right\}$ ) of $\left\{\hat{u}_{j}\right\}$ converging weakly in $H^{s+1}$ on $U$. By compactness, $\hat{u}_{j}$ converges in any weaker norm, say, $L^{2}$ 
norm $|\cdot|_{0}$. By (3.21) with $U, V$ replaced by $U^{\prime}, U\left(U^{\prime}\right.$ being a smaller disc centered at $p$, compactly contained in $U$ ) resp., we learn that $\hat{u}_{j}$ is Cauchy in $H^{s+1}$ on $U^{\prime}$. Therefore $\hat{u}_{j}$ converges in $H^{s+1}$ on $U^{\prime}$. Since $\hat{N}$ is compact, we can pick up a finite number of $\operatorname{such}\left(U^{\prime}, p\right)$ to cover $\hat{N}$. Let $p_{i}$ 's denote such points and $u_{j, U_{i}^{\prime}}$ denote corresponding $u_{j}$ on $U_{i}^{\prime}$. We adjust $a_{j}$ and $\hat{u}_{j}$ to be $a_{j}=\max _{i} u_{j, U_{i}^{\prime}}\left(p_{i}\right)$ and $\hat{u}_{j, U_{i}^{\prime}}=a_{j}^{-1} u_{j, U_{i}^{\prime}}$. Thus $\hat{u}_{j, U_{i}^{\prime}}\left(p_{i}\right)<1$ so that our previous argument still works for all $i$. Now it is easy to pick a subsequence of $m_{a_{j}^{-1}} \phi_{j}$, which converges in $H^{s+1}$ on each $U_{i}^{\prime}$.

Consider the action of $\operatorname{Bdiff} f_{0}^{s+1}$ or $\mathfrak{B}^{s+1}$ on $\widetilde{P}_{i c_{s}}$. First we describe the tangent space of the orbit passing through a given element $\hat{J}$ in $\widetilde{P}_{i c}$. Push $\hat{J}$ down to a complex structure $\hat{c}$ on $\hat{N}$. Take a local holomorphic coordinate $z$ of $\hat{N}$ for $\hat{c}$. Take a local trivialization $(z, w)$ of $\hat{L}$ so that $\partial_{w}$ and $Z_{1}=\partial_{z}+b(z, \bar{z}) \bar{w} \partial_{\bar{w}}$ form a basis of the type $(1,0)$ tangent vectors with respect to $\hat{J}$. (note that $\hat{J}\left(\partial_{z}\right)=i \partial_{z} \bmod \partial_{w}$ and $\partial_{\bar{w}}$, and $Z_{1}$ is $\mathbb{C}^{*}$-invariant) Want to find another trivialization $\widetilde{z}=z, \widetilde{w}=\lambda(z, \bar{z}) w$ so that $\partial_{w}=\lambda \partial_{\widetilde{w}}$ and $Z_{1}=\partial_{\widetilde{z}}\left(\bmod \partial_{w}\right)$. The chain rule tells us that

$$
\partial_{z}=\partial_{\bar{z}}+\frac{\partial \bar{\lambda}}{\partial z}(\bar{\lambda})^{-1} \bar{w} \partial_{\bar{w}}\left(\bmod \partial_{w}\right)
$$

so $\lambda$ has to satisfy the following $\bar{\partial}$-equation:

$$
\frac{\partial \log \lambda}{\partial \bar{z}}=-\bar{b}
$$

But it is easy to solve the above equation locally. ( $\lambda$ is in $H^{s+1}$ if $b$ is in $\left.H^{s}\right)$ Therefore we have a trivialization $(\widetilde{z}, \widetilde{w})$ of $\hat{L}$, holomorphic with respect to $\hat{J}$, i.e. $\left\{\partial_{\widetilde{z}}, \partial_{\widetilde{w}}\right\}$ forms a basis of type $(1,0)$ tangent vectors relative to $\hat{J}$. Now use $(z, w)$ instead of $(\widetilde{z}, \widetilde{w})$ to denote a trivialization of $\hat{L}$, holomorphic with respect to $\hat{J}$, so $\hat{J}=i\left(d z \otimes \partial_{z}+d w \otimes \partial_{w}\right)+$ conjugate. Let $\phi_{t}$ be a family of $H^{s+1}$ bundle automorphisms of $\hat{L}$. Recall that we write the infinitesimal bundle automorphism $V=\left.\frac{d}{d t}\right|_{t=0} \phi_{t}=v^{1} \partial_{z}+v^{*} w \partial_{w}+$ conjugate. (cf.(3.11)) Compute

$$
\left.\frac{d}{d t}\right|_{t=0} \phi_{t}^{*} \hat{J}=L_{V} \hat{J}=2 i \partial_{\bar{z}} v^{1} d \bar{z} \otimes \partial_{z}+2 i w \partial_{\bar{z}} v^{*} d \bar{z} \otimes \partial_{w}+\text { conjugate. }
$$

Recall that $\tilde{\mathfrak{V}}^{s}$ denote the Hilbert space of all infinitesimal bundle automorphisms with bounded $H^{s}$-norm. Define the first order operator 
$P: \widetilde{\mathfrak{V}}^{s+1} \rightarrow T_{\hat{J}} \widetilde{P}_{i c_{s}}=\mathfrak{E}_{\hat{J}}^{s}$ by

$$
P(V)=L_{V} \hat{J}=2 i v_{, 1}^{1} d \bar{z} \otimes \partial_{z}+2 i v_{,{ }_{,}} w d \bar{z} \otimes \partial_{w}+\text { conjugate }
$$

in trivialization $(z, w)$, where $v_{, \overline{1}}^{1}=\partial_{\bar{z}} v^{1}, v_{, \overline{1}}^{*}=\partial_{\bar{z}} v^{*}$. We want to describe the $L^{2}$ orthogonal subspace of Range $(\mathrm{P})$ in $\mathfrak{E}_{\hat{J}}^{s}$, which is supposed to be the kernel $\operatorname{Ker} P^{*}$ of the adjoint operator $P^{*}$. Since $\hat{L}$ is not compact, we need to define a suitable inner product on $\mathfrak{E}_{\hat{J}}^{s}$ over $\hat{N}$. First observe from (3.7.1) that $E_{1}{ }^{\overline{1}}$ behaves just as a tensor on $\hat{N}$ (under the special coordinate change (3.8)) while $E_{1}{ }^{*}$ does not by (3.7.2). We can adjust $E_{1}{ }^{*}$ to get a tensor by the aid of connection. Let \|\| be a hermitian metric on $\hat{L}$. Let $s(z)$ denote the local holomorphic section of $(\hat{L}, \hat{J})$ given by $z \rightarrow(z, 1)$ locally. Let $\nu=\|s(z)\|^{2}$. The canonical connection associated to \|\| is given by

$$
\nu^{-1} \partial \nu=\Gamma(z, \bar{z}) d z
$$

with $\Gamma=\partial_{z} \log \nu$. The transformation law according to (3.8) goes as follows:

$$
\Gamma=\widetilde{\Gamma} h^{\prime}+g^{\prime} g^{-1} \text {. }
$$

(noting that $g \widetilde{s}=s$ ) Define

$$
E_{1}=E_{1}{ }^{*}+E_{1}{ }^{\overline{1}} \bar{\Gamma}
$$

It is easy to see that $E_{1}=\widetilde{E}_{1} h^{\prime}$, obeying the correct transformation law as a tensor. Let $g=g_{1 \overline{1}} d z d \bar{z}$ be the unique hyperbolic metric on $\hat{N}$ associated to $\hat{c}$. We use $g^{1 \overline{1}}=\left(g_{1 \overline{1}}\right)^{-1}$ or $g_{1 \overline{1}}$ to raise or lower indices. Also denote the volume form of $\mathrm{g}$ by dvol $g$. Now we can define an inner product on $\mathfrak{E}_{\hat{J}}^{s}$ :

$$
\langle E, F\rangle=\int_{\hat{N}}\left\{E_{1}{ }^{\overline{1}} F_{\overline{1}}^{1}+g^{1 \overline{1}} E_{1} F_{\overline{1}}\right\} d v o l_{g} .
$$

Here we have used the expression (3.6) for $E, F$ and (3.24) for $E_{1}, F_{\overline{1}}=\overline{\left(F_{1}\right)}$. Take $E=P(V)$. Comparing (3.6) and (3.23) gives

$$
E_{1}{ }^{\overline{1}}=-2 i v^{\overline{1}}, 1, E_{1}^{*}=-2 i \bar{v}^{*}, 1 .
$$

Here $v^{\overline{1}}=\overline{\left(v^{1}\right)}$ and $u_{, 1}=\partial_{z} u$. Define

$$
v=v^{*}+v^{1} \Gamma \text {. }
$$

Easy to check that $v$ is independent of the choice of holomorphic trivializations. Hence $v$ defines a global function on $\hat{N}$. Recall that $c_{1}(=-m)$ 
denotes the first Chern number of $\hat{L}$. Let $\mu=(1 / 4) c_{1}(\operatorname{genus}(\hat{N})-1)$. For a special choice of \|\| according to Proposition 2.1 relative to $\hat{J}$, we compute

$$
E_{1}=-2 i\left[\bar{v}_{, 1}+\mu v_{1}\right]
$$

where $v_{1}=v^{\overline{1}} g_{1 \overline{1}}$ and we have used $-g^{1 \overline{1}} \bar{\Gamma}_{, 1}=\mu$. Substituting (3.26),(3.28) in (3.25) and using integration by parts gives

$$
\langle P(V), F\rangle=2 i \int_{\hat{N}}\left\{v^{1}\left(-F_{1}{ }^{\overline{1}}, \overline{1}+\mu F_{1}\right)-v F_{1, \overline{1}} g^{1 \overline{1}}\right\} d v o l_{g}+\text { conjugate. }
$$

(note that since $g$ is Kahler, the usual derivative of $v^{1}$ along $\bar{z}$-direction coincides with its covariant derivative. Hereafter for a tensor $T$ on $\hat{N}, T_{, 1}\left(T_{, 1 \overline{1}}\right.$ and so on, resp.) means the covariant derivative of $T$ in the $z$-direction $(z \bar{z}$-direction and so on, resp.))

The above formula suggests a suitable inner product on $\widetilde{\mathfrak{V}}^{s}$ for our purpose. Namely, we define

$$
\langle V, U\rangle=\int_{\hat{N}}\left[g_{1 \overline{1}} v^{1} u^{\overline{1}}+v \bar{u}\right] d v o l_{g}+\text { conjugate }
$$

for $V=2 \operatorname{Re}\left[v^{1} \partial_{z}+v^{*} w \partial_{w}\right], U=2 \operatorname{Re}\left[u^{1} \partial_{z}+u^{*} w \partial_{w}\right]$ locally and $v, u$ being global functions defined by (3.27). Define the adjoint operator $P^{*}$ of $P$ : $\mathfrak{E}_{\hat{J}}^{s} \rightarrow \widetilde{\mathfrak{V}}^{s-1}$ so that

$$
\langle P(V), F\rangle=\left\langle V, P^{*}(F)\right\rangle .
$$

Then it follows from (3.29),(3.30) and (3.27) that locally

$$
\begin{aligned}
P^{*}(F)= & 2 i\left(F_{\overline{1}}{ }^{1}, 1-\mu F_{\overline{1}}\right) g^{1 \overline{1}} \partial_{z}+2 i\left[F_{\overline{1}}{ }^{*}, 1+F_{\overline{1}}{ }^{*} \Gamma\right. \\
& \left.+F_{\overline{1}}{ }^{1}\left(\Gamma_{, 1}+\Gamma^{2}\right)\right] g^{1 \overline{1}} w \partial_{w}+\text { conjugate. }
\end{aligned}
$$

If we represent $V$ by the pair $\left(v^{1}, v\right)$ and $E$ by the pair $\left(E_{\overline{1}}{ }^{1}, E_{\overline{1}}\right)$. Then we can write $P(V)$ and $P^{*}(F)$ as follows:

$$
\begin{aligned}
P(V) & =2 i\left(v^{1}{ }_{, \overline{1}}, v_{, \overline{1}}+\mu v_{\overline{1}}\right) \\
P^{*}(F) & =2 i\left(g^{1 \overline{1}}\left(F_{\overline{1}}^{1}{ }_{, 1}-\mu F_{\overline{1}}\right), g^{1 \overline{1}} F_{\overline{1}, 1}\right) .
\end{aligned}
$$

Let $\Delta_{\hat{J}}=P^{*} P$. By (3.31), we compute

$$
\Delta_{\hat{J}}(V)=-4\left(g^{1 \overline{1}}\left(v_{, \overline{1} 1}^{1}-\mu\left(v_{, \overline{1}}+v_{\overline{1}} \mu\right)\right), g^{1 \overline{1}}\left(v_{, \overline{1} 1}+\mu v_{\overline{1}, 1}\right)\right) .
$$

The leading term of $\Delta_{\hat{J}}(V)$ is $-4\left(\Delta_{g} v^{1}, \Delta_{g} v\right)$ where the Laplacian $\Delta_{g}=$ $g^{1 \overline{1}} \partial^{2} / \partial z d \bar{z}$. Thus $\Delta_{\hat{J}}$ is a second order self-adjoint elliptic operator defined on $\widetilde{\mathfrak{V}}^{s}$. 
Lemma 3.6. Suppose $\hat{J} \in \widetilde{P}_{i c_{s+1}}$. Then there is an $L^{2}$-orthogonal splitting

$$
\mathfrak{E}_{\hat{J}}^{s}=\operatorname{Ker} P^{*}+P\left(\widetilde{\mathfrak{V}}^{s+1}\right) \text {. }
$$

Proof. Given $E$ in $\mathfrak{E}_{\hat{J}}^{s}$. It is easy to see that $P^{*}(E)$ is orthogonal to the kernel $\operatorname{Ker} \Delta_{\hat{J}}$ of $\Delta_{\hat{J}}$ since $\operatorname{Ker} \Delta_{\hat{J}}=\operatorname{Ker} P$. Therefore by the standard elliptic theory we can solve the equation $\Delta_{\hat{J}}(V)=P^{*}(E)$ for $V$ in $H^{s+1}$. Now set $E_{0}=E-P(V)$. It is obvious that $E_{0}$ is in $\operatorname{Ker} P^{*}$.

We remark that elements in $\operatorname{Ker} P^{*}$ are all smooth by the elliptic regularity. (note that $g_{1 \overline{1}}$ has the same regularity as $\hat{J}$ does $[\mathrm{Tr}]$ )

Moreover the dimension of $\operatorname{KerP}{ }^{*}$ is finite. We compute it as follows. First an element $F$ in $K e r P^{*}$ satisfies a system of linear equations:

$$
\begin{aligned}
F_{1}{ }^{\overline{1}} \overline{1}-\mu F_{1} & =0 \\
F_{1, \overline{1}} & =0
\end{aligned}
$$

by (3.31). Solutions $F_{1}$ for (3.32b) consist of all holomorphic $(1,0)$-forms $F_{1} d z$ on $\hat{N}$, denoted $H^{1,0}$. Let $Q(\hat{N})$ denote the space of holomorphic quadratic differentials on $\hat{N}$. By (3.32), the projection map from $\operatorname{Ker} P^{*}$ onto $H^{1,0}$ has the kernel equal to $Q(\hat{N})$. From the basic linear algebra we learn that

$$
\operatorname{dim} \operatorname{Ker} P^{*}=\operatorname{dim} Q(\hat{N})+\operatorname{dim} H^{1,0} .
$$

On the other hand, $Q(\hat{N})$ is known to describe the infinitesimal Teichmuller space whose dimension is $6 g-6$ by the Riemann-Roch theorem (e.g. [Tr]) while $\operatorname{dim} H^{1,0}$ is the same as that of the so-called Picard variety in the Riemann surface theory, which is known to be $2 g$. Therefore

$$
\operatorname{dim} K \operatorname{Ker} P^{*}=6 g-6+2 g=8 g-6 .
$$

Lemma 3.7. Given $\hat{J}$ in $\widetilde{P}_{i c}$, there exists a local smooth submanifold $\mathfrak{S}$ of $\widetilde{P}_{i c_{s}}$ of dimension $8 g-6$ passing through $\hat{J}$ with the tangent space equal to KerP* at $\hat{J}$. Moreover, $\mathfrak{S}$ consists of only smooth elements.

Proof. Consider the map $\Phi_{\hat{J}}: K e r P^{*} \rightarrow \widetilde{P}_{i c_{s}}$. (see (3.9)) It is easy to see that $\Phi_{\hat{J}}$ is smooth and its functional derivative at 0 is the inclusion map from $\operatorname{Ker} P^{*}$ into $\mathfrak{E}_{\hat{J}}^{s}$, which is surely injective and splits by Lemma 3.6. Therefore $\Phi_{\hat{J}}$ is a smooth immersion at 0 . That is to say, there exists a 
small neighborhood $U$ of 0 such that $\mathfrak{S}=\Phi_{\hat{J}}(U)$ is a smooth submanifold of $\widetilde{P}_{i c_{s}}$ with dimension $8 g-6$ by (3.33). Note that $\hat{J}$ is smooth and elements in $\operatorname{Ker} P^{*}$ are smooth as remarked previously. Thus $\mathfrak{S}$ consists of only smooth elements.

Now let $\Xi: \mathfrak{B}^{s+1} \times \mathfrak{S} \rightarrow \widetilde{P}_{i c_{s}}$ denote the action of $\mathfrak{B}^{s+1}$ on $\mathfrak{S}$ by the pullback. Observe that $\Xi$ is smooth and

$$
D \Xi(i d, \hat{J}): \mathfrak{V}^{s+1} \times K \operatorname{Ker} P^{*} \rightarrow \mathfrak{E}_{\hat{J}}^{s}
$$

is given by $D \Xi(i d, \hat{J})([X], E)=L_{X} \hat{J}+E=P(X)+E$. If $L_{X} \hat{J}=0$, then $X$ is an infinitesimal bundle automorphism fixing $\hat{J}$. Thus $[X]=0$ by Proposition 3.4 and hence $D \Xi(i d, \hat{J})$ is a continuous linear isomorphism by further using Lemma 3.6 and noting that $\Delta_{\hat{J}}$ is elliptic. Therefore $\Xi$ is a local diffeomorphism by the inverse function theorem on Banach spaces. We have shown the existence of "local slices":

Proposition 3.8. There exist neighborhoods $W$ of $\hat{J}$ in $\widetilde{P}_{i c_{s}}, U$ of id in $\mathfrak{B}^{s+1}$ and $V$ of $\hat{J}$ in $\mathfrak{S}$ such that $\Xi: U \times V \rightarrow W$ is a diffeomorphism.

Now using freeness and properness of our $\mathfrak{B}^{s+1}$ action (Propositions $3.4,3.5$ ) plus the existence of "local slices" (Proposition 3.8), we can equip our quotient space $\widetilde{P}_{i c} / \mathfrak{B}$ with smooth manifold structure by a standard argument. (e.g. section 2.4 in [Tr] ) Recall that we denote $\widetilde{P}_{i c} / \mathfrak{B}$ by $P_{i c}^{t}$.

Theorem 3.9. $P_{i c}^{t}$ is a smooth manifold of dimension $8 g-6$.

Proof. First we show the existence of "slices": that is to say, if we take the slice $\mathfrak{S}$ to be sufficiently small, then each orbit of $\mathfrak{B}$ passing through $\mathfrak{S}$ intersects $\mathfrak{S}$ at exactly one point, i.e. $\phi^{*} J$ in $\mathfrak{S}$ with $J$ in $\mathfrak{S}$ implies $\phi=i d$. Suppose this is not true. Then there are sequences $\phi_{j}$ in $\mathfrak{B}$ and $J_{j}$ in $\mathfrak{S}$ such that $J_{j}$ and $\phi_{j}^{*} J_{j}$ converge to $\hat{J}$ in $H^{s}$ while all $\phi_{j}$ 's keep ontside some fixed $H^{s+1}$ neighborhood of $i d$ in $\mathfrak{B}$ in view of Proposition 3.8. (we equip $\widetilde{P}_{i c}, \mathfrak{B}$ with the $H^{s}, H^{s+1}$ topologies, resp.) By Proposition 3.5 (properness) there exists a subsequence of $\phi_{j}$, which converges to $\phi$ in $H^{s+1}$. It follows that $\phi^{*} \hat{J}=\hat{J}$ and then $\phi=i d$ by Proposition 3.4 (freeness), contrary to $\phi_{j}$ 's sitting outside some neighborhood of $i d$. Thus we can take the slices as coordinate charts (instead of their tangent spaces). It is easy to see by Proposition 3.8 that the transition function is smooth. 
Proof of Theorem $C$. We will introduce a natural complex structure on $P_{i c}^{t}$. First there is a canonical way to define an almost complex structure $\Theta$ on $\widetilde{P}_{i c}:$ for $J$ in $\widetilde{P}_{i c}, E$ in $\mathfrak{E}_{J}$

$$
\Theta_{J}(E)=J \circ E .
$$

It is easy to verify that $J \circ E$ is still sitting in $\mathfrak{E}_{J}$. Let $\pi: \widetilde{P}_{i c} \rightarrow P_{i c}^{t}$ be the natural projection. From our previous argument $\left(\pi, \mathfrak{B}, \widetilde{P}_{i c}, P_{i c}^{t}\right)$ is a (weak) principal $\mathfrak{B}$-bundle in the sense of [Tr], p.54. (note that the right action of $\mathfrak{B}$ on $\widetilde{P}_{i c}$ is given by pulling back) It is straightforward that $\Theta$ is $\mathfrak{B}$-invariant (cf. p.88 in [Tr]), and $\Theta$ maps "vertical" vectors to "vertical" vectors: (see p.86 in [Tr] for the definition) since each $\mathrm{J}$ in $\widetilde{P}_{i c}$ is integrable by Proposition 3.1, the associated Nijenhuis tensor vanishes. It follows that $\Theta_{J}\left(L_{X} J\right)=$ $J L_{X} J=L_{J X} J$ (cf. p.88 in [Tr]), so $\Theta$ makes $\left(\pi, \mathfrak{B}, \widetilde{P}_{i c}, P_{i c}^{t}\right)$ into an almost complex principal $\mathfrak{B}$-bundle. (see Definition 4.1.4 on p.86 in [Tr]) Next we note that the Lie bracket of two vector fields on $\widetilde{P}_{i c}$ can be defined as in [Tr], p.85: instead of using projections, we view $D Y(J) X(J)=d /\left.d t\right|_{t=0} Y(J(t))$ with $J(0)=J, J^{\prime}(0)=X(J)$; verify $D Y(J) X(J)-D X(J) Y(J)$ is in $\mathfrak{E}_{J}$ for $X(J), Y(J)$ in $\mathfrak{E}_{J}$ by observing that an element $E$ in $\mathfrak{E}_{J}$ can be described by the following conditions:

$$
\begin{aligned}
E \circ J+J \circ E & =0 \\
m_{\rho}^{*} E & =E \\
E(v) & =0 \text { for } v \text { tangent to fibres of } \hat{L} .
\end{aligned}
$$

Now we can define the Nijenhuis tensor $N(\Theta)$ of $\Theta$ on $\widetilde{P}_{i c}$ as usual. Then a direct computation as shown in [Tr], p.88 yields $N(\Theta)=0$. By Theorem 4.1.2 in [Tr], the almost complex structure $J_{p i c}$ on $P_{i c}^{t}$ induced from $\Theta$ on $\widetilde{P}_{i c}$ has the vanishing Nijenhuis tensor. Since $P_{i c}^{t}$ is a finite dimensional manifold, $J_{p i c}$ is integrable, i.e. there exists a complex structure on $P_{i c}^{t}$ whose associated almost complex structure is $J_{p i c}$ by the Newlander-Nirenberg theorem.

\section{A supporting manifold of $\mathfrak{S}^{t}$ and properness of the contact action.}

Recall (cf. section 1) that $\mathfrak{S}^{t}$ is the quotient space of $\widetilde{\mathfrak{S}}$ modulo $C_{\hat{H}}^{0}$. Here $\widetilde{\mathfrak{S}}$ denotes the space of all smooth spherical CR manifolds $(\hat{S}, \hat{H}, J)$ with $J$ oriented and compatible with $\hat{H}$ and $C_{\hat{H}}^{0}$ denotes the identity component of the orientation-preserving smooth contact diffeomorphism group $C_{\hat{H}}$ relative 
to $\hat{H}$. In this section we will parametrize a local "supporting" space of $\mathfrak{S}^{t}$ and show the properness of the $C_{\hat{H}}^{0}$ action.

We will work with the aid of anisotropic Folland-Stein spaces. For $F$ a vector bundle over a closed contact manifold $(M, H)$ and $k$ a nonnegative integer, let $S_{k}(F)$ denote the $L^{2}$ Folland-Stein space of sections of $F$. ([FS], p.241 in [CL1]) If the bundle is clear from the context, we simply use the notation $S_{k}$ instead of $S_{k}(F)$, and a norm on $S_{k}$ is denoted by $|\cdot|_{k}$. Let $\widetilde{\mathfrak{S}}^{k}$ denote the completion of $\widetilde{\mathfrak{S}}$ under the norm $|\cdot|_{k}$ for a fixed smooth background contact manifold $(\hat{S}, \hat{H})$. Let $\mathfrak{J}^{k}(\mathfrak{J}$,resp.) denote the space of all oriented compatible $S_{k}\left(C^{\infty}\right.$,resp.) CR structures on $(\hat{S}, \hat{H})$ or a general contact manifold $(M, H)$ depending on the context. (note that these $\mathrm{CR}$ structures are sections of the endomorphism bundle End $(\hat{H}))$

Lemma 4.1. Suppose $\operatorname{dim} M=3$. For $k \geq 6$, (a) $S_{k}$ is an algebra; (b) Let $f$ be a smooth function on nonnegative real numbers. Then $f \circ h$ is still in $S_{k}$ for nonnegative $S_{k}$ function $h$.

Proof. . (a) is known. (e.g. [BD]) (b) is probably also known. We prove it by induction on $k$. Computing the derivative of $f \circ h$ in some contact direction give the derivative of $f$ composed with $h$ times the derivative of $h$ in that direction, so induction hypothesis on $k-1$ plus (a) implies the derivative of $f \circ h$ is in $S_{k-1}$. Hence $f \circ h$ is in $S_{k}$, so to complete the proof we have to check the starting case $k=6$. But it is straightforward by observing that $S_{6}$ is contained in $S_{1}^{12}$ or $S_{2}^{8}$ and $S_{3}$ is contained in $C^{0}$, etc.. (see e.g. Theorem 4.17 , Corollary 5.16 in [Fo])

Lemma 4.2. $\mathfrak{J}^{k}$ is a Hilbert manifold for large $k$, say, $k \geq 6$.

Proof. In [CL1], we parametrize $\mathfrak{J}^{k}$ for $k=\infty$, i.e. in the smooth category by a map $\Phi_{J}$ given by

$$
\Phi_{J}(E)=E_{0} J+E, \quad E_{0}=\left(1+(1 / 2) \operatorname{Tr}\left(E^{2}\right)\right)^{1 / 2} .
$$

(p.228, Lemma 2.3 in [CL1]) Suppose $E$ is in $S_{k}$. (more precisely $S_{k}\left(\mathfrak{E}_{J}\right)$ ) By Lemma 4.1(a) $h=E_{1}{ }^{\overline{1}} E_{\overline{1}}{ }^{1}$ is in $S_{k}$. Take $f(x)=(1+x)^{1 / 2}$. By Lemma 4.1(b) $E_{0}$ is in $S_{k}$. Therefore $\Phi_{J}$ preserves $S_{k}$ spaces, so does $\pi_{J}$. Thus we can still use $\Phi_{J}$ or $\pi_{J}$ to parametrize $\mathfrak{J}^{k}$ modelled on $S_{k}\left(\mathfrak{E}_{J}\right)$.

Hereafter throughout this paper we will assume that $k \geq 6$ unless specified otherwise. We know that a CR structure $J$ being spherical is char- 
acterized by the vanishing of the Cartan tensor $Q_{J}$. (p.227 in [CL1]) The linearization $D Q_{J}$ is subelliptic when restricted to $\operatorname{Ker} B_{J}$. (in view of Lemma 3.3 and Proposition 3.1 in [CL1]) When working in the FollandStein category, it is enough to still require the reference CR structure to be smooth for our purpose.

Lemma 4.3. For a smooth spherical $\hat{J}$ in $\mathfrak{J}^{k}$ (and an auxiliary smooth contact form), we have the following $L^{2}$-orthogonal decomposition:

$$
S_{k}\left(\mathfrak{E}_{\hat{J}}\right)=\operatorname{Ker}_{k} D Q_{\hat{J}}+D Q_{\hat{J}}\left(S_{k+4}\left(\mathfrak{E}_{\hat{J}}\right)\right)
$$

where $K r_{k}$ means elements in the kernel and also in $S_{k}$.

Proof. Differentiating the Bianchi identity $B_{J} Q_{J}=0$ in Proposition 3.1 of [CL1] at $\hat{J}$ in the direction E implies $D Q_{\hat{J}}(E)$ belongs to the kernel of $B_{\hat{J}}$. (note that $Q_{\hat{J}}=0$ ) On the other hand, for $E$ in $\operatorname{Ker}_{k} B_{\hat{J}}$, we have

$$
D Q_{\hat{J}}(E)=-(1 / 24) L_{a}^{*} L_{a}(E)+\text { terms of lower weight }
$$

with $a=4+i \sqrt{3}$ according to Lemmas 3.3, 3.2 in [CL1]. For $a$ not an odd integer, $L_{a}$ is a subelliptic operator of weight 2 , i.e. satisfies the estimate (4.2) in [CL1], so restricted to $\operatorname{Ker}_{k} B_{\hat{J}}, D Q_{\hat{J}}$ is a subelliptic operator of weight 4 according to the above formula, i.e. earns four derivatives in contact directions and we have the $L^{2}$-orthogonal decomposition for $D Q_{\hat{J}}$ :

$$
\operatorname{Ker}_{k} B_{\hat{J}}=K \operatorname{KerD} Q_{\hat{J}}+D Q_{\hat{J}}\left(\operatorname{Ker}_{k+4} B_{\hat{J}}\right) .
$$

Here $\operatorname{Ker} D Q_{\hat{J}}$ consists of smooth elements since $D Q_{\hat{J}}$ is subelliptic, hence hypoelliptic when restricted to $\operatorname{Ker}_{k} B_{\hat{J}}$. We also have the $S_{k}$ version of Proposition 2.4 in [CL2]:(note that notation $D_{J}$ in [CL2] $=B_{J}^{\prime}$ in [CL1])

$$
S_{k}\left(\mathfrak{E}_{\hat{J}}\right)=\operatorname{Ker}_{k} B_{\hat{J}}+B_{\hat{J}}^{\prime}\left(S_{k+2}\right)
$$

basically because $\Delta_{\hat{J}}=B_{\hat{J}} B_{\hat{J}}^{\prime}$ is a subelliptic operator of weight 4 by Lemma 2.1 in [CL2]. Since each element in the range of $B_{\hat{J}}^{\prime}$ is an infinitesimal contact orbit at $\hat{J}$ and $Q_{J}$ equals 0 for $J$ in the contact orbit of $\hat{J}, D Q_{\hat{J}}$ vanishes on $B_{\hat{J}}^{\prime}\left(S_{k+2}\right)$. Therefore we can combine (4.3) and (4.2) to get (4.1).

Take a smooth $\hat{J}$ in $\widetilde{\mathfrak{S}}$ and choose an auxiliary smooth contact form $\hat{\theta}$. There is a local slice $\mathfrak{S}$ of $\mathfrak{J}$ passing through $\hat{J}$ by Theorem A of [CL2], defined by $\Phi_{\hat{J}}\left(\operatorname{Ker} B_{\hat{J}}\right)\left(\right.$ note $\left.B_{\hat{J}}=D_{\hat{J}}^{*}\right)$ for elements in Ker $B_{\hat{J}}$ with small 
$|\cdot|_{5, \infty}$ norm. By the Sobolev lemma for our anisotropic spaces (e.g., (4.17), (5.15) in [F]), we have $S_{k} \subset S_{8-4 / q} \subset S_{6}^{q} \subset \Gamma_{6-4 / q} \subset S_{5}^{\infty}$ for $k \geq 8$. Thus taking elements of small $S_{k}$ norm, $k \geq 8$, in $K e r B_{\hat{J}}$ and then sending them to $\mathfrak{J}^{k}$ through $\Phi_{\hat{J}}$, we obtain an $S_{k}$ slice $\mathfrak{S}_{(k)}$ passing through $\hat{J}$. Consider the map $\mathfrak{Q}: \mathfrak{S}_{(k)} \rightarrow D Q_{\hat{J}}\left(S_{k}\left(\mathfrak{E}_{\hat{J}}\right)\right.$, defined by

$$
\mathfrak{Q}(J)=\pi\left(Q_{J}\right) .
$$

Here $Q_{J}$ is the Cartan tensor of $(J, \hat{\theta}), \pi$ is the composition of the orthogonal projection $\pi_{\hat{J}}: S_{k-4}(\operatorname{End}(\hat{H})) \rightarrow S_{k-4}\left(\mathfrak{E}_{\hat{J}}\right)$ (p.228 in [CL1]) and the projection: $S_{k-4}\left(\mathfrak{E}_{\hat{J}}\right) \rightarrow D Q_{\hat{J}}\left(S_{k}\left(\mathfrak{E}_{\hat{J}}\right)\right)$ according to (4.1).

Proposition 4.4. $\mathfrak{Q}^{-1}(0)$ is a smooth finite dimensional submanifold of $\mathfrak{S}_{(k)}$ for $k \geq 10$ near a smooth $\hat{J}$.

Proof. It is easy to see that $\pi$ is smooth and since $Q_{J}$ is of type 4 (pp.249-250 in [CL1]), the map: $J \in S_{k} \rightarrow Q_{J} \in S_{k-4}$ for $k \geq 10$ is smooth. (note that $S_{k}$ forms an algebra for $k \geq 6$ by Lemma 4.1) Therefore $\mathfrak{Q}$ is smooth. We compute

$$
\begin{aligned}
D \mathfrak{Q}(\hat{J})(E) & =D \pi(0) D Q_{\hat{J}}(E) \\
& =\pi\left(D Q_{\hat{J}}(E)\right)=D Q_{\hat{J}}(E)
\end{aligned}
$$

for $E$ in $S_{k}\left(\mathfrak{E}_{\hat{J}}\right)$. From (4.4) it is clear that $D \mathfrak{Q}(\hat{J})$ is surjective. Furthermore, the kernel of $D \mathfrak{Q}(\hat{J})$ is the same as the kernel of $D Q_{\hat{J}}$, which splits according to (4.1). Thus by the inverse function theorem $\mathfrak{Q}$ is a submersion at $\hat{J}$ (Proposition 2 on p.27 in [La]), so $\mathfrak{Q}^{-1}(0)$ has a smooth submanifold structure near $\hat{J}$. Moreover, finite-dimensionality follows from subellipticity of $D Q_{\hat{J}}$ restricted to $\operatorname{Ker} B_{\hat{J}}$.

We will use $\mathfrak{Q}^{-1}(0)$ as a "supporting" background manifold to prove $\mathfrak{S}_{0}^{t}$ (an open connected subspace of $\mathfrak{S}^{t}$; see section 5 ) is a smooth manifold. First we will show the properness of the contact action in the negative pseudohermitian curvature case. Let $(M, H)$ be a smooth, closed, oriented, contact 3-manifold. Let $S_{k}(M, \mathbb{R})$ denote the space of all real-valued $S_{k}$ functions on $(M, H)$.

Lemma 4.5. The pseudohermitian curvature $R_{J, \theta}$ belongs to $S_{k-2}(M, \mathbb{R})$ for $J, \theta$ in $S_{k}$ with $k \geq 8$. 
Proof. Take a smooth contact form $\hat{\theta}$. Write $\theta=u^{2} \hat{\theta}$ for positive $u$ in $S_{k}(M, \mathbb{R})$. The transformation law reads

$$
4 \Delta_{b} u+R_{J, \hat{\theta}} u-R_{J, \theta} u^{3}=0 .
$$

([JL]) Here the negative sublaplacian $\Delta_{b}$ is defined with respect to $(J, \hat{\theta})$. Suppose $J$ is in $S_{k}$. Then it is easy to see that $\Delta_{b} u$ is in $S_{k-2}$ in view of Lemma 4.1 if we write $J$ with respect to a smooth $\hat{J}$ as in p.249 of [CL1] and apply formulas on pp. 249-250 of [CL1] to express $\Delta_{b} u$. Moreover $R_{J, \hat{\theta}}$ is in $S_{k-2}$ since $R_{J, \hat{\theta}}$ is of type 2 as shown in the following transformation formula:

$$
\begin{aligned}
R_{J, \hat{\theta}}= & R_{\hat{J}, \hat{\theta}}+\frac{1}{2} i\left(v_{\overline{1}}{ }^{1}, 0 v_{1}{ }^{\overline{1}}-v_{1}{ }^{\overline{1}}{ }_{, 0} v_{\overline{1}}{ }^{1}\right) \\
& -v_{0}\left(v_{0,1 \overline{1}}+v_{0, \overline{1} 1}+v_{11, \overline{1} \overline{1}}+v_{\overline{1} \overline{1}, 11}\right) \\
& -v_{\overline{1} \overline{1}}\left(v_{0,11}+v_{11, \overline{1} 1}\right)-v_{11}\left(v_{0, \overline{1} \overline{1}}+v_{\overline{1} \overline{1}, 1 \overline{1}}\right)-2\left|v_{0,1}+v_{11, \overline{1}}\right|^{2}
\end{aligned}
$$

(see pp.249-250 in [CL1] where we did not give the above formula precisely) Now from (4.5) $R_{J, \theta}$ is therefore in $S_{k-2}$ in view of Lemma 4.1 .

Lemma 4.6. Let $(M, H)$ be a smooth, closed, oriented, contact 3-manifold. Suppose the pseudohermitian curvature $R_{\hat{J}, \hat{\theta}}=-1$ for some smooth $(\hat{J}, \hat{\theta})$ on $(M, H)$. Then for any $J$ in $\mathfrak{J}^{k}, k \geq 8$, there exists a uniquely determined $S_{k}$ contact form $\theta$ with $R_{J, \theta}=-1$.

Proof. Consider the map $\mathfrak{R}: \mathfrak{J}^{k} \times\left\{S_{k}\right.$ contact forms $\} \rightarrow S_{k-2}(M, \mathbb{R})$ defined by

$$
\Re(J, \theta)=R_{J, \theta} .
$$

(well defined by Lemma 4.5) The map $\Re$ is smooth in view of (4.5) and (4.6). Differentiating $\mathfrak{R}$ at $(\hat{J}, \theta)$ in the direction $\left(J^{\prime}, \theta^{\prime}\right)=(2 E, 2 h \hat{\theta})$ gives

$$
\begin{aligned}
D \Re(\hat{J}, \hat{\theta})(2 E, 2 h \hat{\theta})= & i\left(E_{11, \overline{1} \overline{1}}-E_{\overline{1} \overline{1}, 11}\right)-\left(A_{11} E_{\overline{1} \overline{1}}+A_{\overline{1} \overline{1}} E_{11}\right) \\
& +4 \Delta_{b} h-2 R_{\hat{J}, \hat{\theta}} h
\end{aligned}
$$

according to (2.20) in [CL1] and (5.15) in [Lee]. Since $R_{\hat{J}, \hat{\theta}}=-1,4 \Delta_{b}-$ $2 R_{\hat{J}, \hat{\theta}}=4 \Delta_{b}+2 I d$ is invertible. It follows that $D \mathfrak{R}(\hat{J}, \hat{\theta})$ is surjective. Moreover it is easy to see that the kernel $(D \Re(\hat{J}, \hat{\theta}))^{-1}(0)$ and the space $\{(0,2 h \hat{\theta})\}$ span the tangent space of the domain at $(\hat{J}, \hat{\theta})$ and have only $(0,0)$ in their intersection. That is to say, $(D \Re(\hat{J}, \hat{\theta}))^{-1}(0)$ splits. Therefore 
$\mathfrak{R}$ is a submersion at $(\hat{J}, \hat{\theta})$. (Prop.2 on p.27 in [La]) Thus $\mathfrak{R}^{-1}(-1)$ has a submanifold structure near $(\hat{J}, \hat{\theta})$ and it projects onto a neighborhood of $\hat{J}$ in $\mathfrak{J}^{k}$.

On the other hand, suppose $R_{J_{j}, \theta_{j}}=-1$ for a sequence of $\operatorname{smooth}\left(J_{j}, \theta_{j}\right)$. (note that $\theta_{j}$ is uniquely determined by $J_{j}$ by Prop. 2.2) If $J_{j}$ tends to $J$ in. $S_{k}$, we claim that $\theta_{j}$ tends to $\theta$ in $S_{k}$ too so that $R_{J, \theta}=-1$. Write $\theta_{j}=u_{j}^{2} \hat{\theta}$ for positive $u_{j}$. Then $u_{j}$ satisfies the equation (4.5) with $(J, \theta)$ replaced by $\left(J_{j}, \theta_{j}\right) . \quad R_{J_{j}, \theta_{j}}=-1$ implies $R_{J_{j}, \hat{\theta}}$ must be negative by the maximum principle. Moreover apply the maximum principle to the equation (4.5) where $u_{j}$ is a maximum, hence (negative sublaplacian) $\Delta_{b} u_{j} \geq 0$. Since both $R_{J_{j}, \hat{\theta}}$ and $R_{J_{j}, \theta_{j}}$ are negative, we get the uniform $C^{0}$ estimate of $u_{j}$ :

$$
\max u_{j} \leq\left(-R_{J_{j}, \hat{\theta}}\right)_{\max }^{1 / 2} \leq C
$$

for a constant $C$ independent of $j$ in view of (4.6). Similarly applying the maximum principle at the minimum of $u_{j}$, we obtain

$$
0 \leq c \leq\left[\left(-R_{J_{j}, \hat{\theta}}\right)_{\min }\right]^{\frac{1}{2}} \leq \min u_{j}
$$

for a positive constant $c$ independent of $j$. Let $\Delta_{b}$ and $\Delta_{b(j)}$ denote the negative sublaplacians with respect to $(J, \hat{\theta})$ and $\left(J_{j}, \hat{\theta}\right)$ respectively. Using those formulas on pp.249-250 in [CL1], we have the following estimate: given a small $\epsilon>0$,

$$
\left|\Delta_{b(j)} u-\Delta_{b} u\right|_{k-2} \leq \epsilon|u|_{k}
$$

for $j$ large and $u$ in $S_{k}$. For $J$ in $S_{k}$ the difference between $\Delta_{b}$ and the corresponding operator on the Heisenberg group is small for a small region on $M$ in the sense of (4.10). By a standard argument (absorbing the right side of (4.10) and using a partition of unity for compact $M$ ), we still have the subelliptic estimate for $\Delta_{b}$ :

$$
\left|u_{j}\right|_{k} \leq C\left(\left|\Delta_{b} u_{j}\right|_{k-2}+\left|u_{j}\right|_{0}\right) .
$$

Write $\Delta_{b} u_{j}=\Delta_{b(j)} u_{j}+\left(\Delta_{b}-\Delta_{b(j)}\right) u_{j}$ and substitute in (4.11). Using (4.10), absorbing the right side to the left and applying the equation (4.5) to $\Delta_{b(j)} u_{j}$, we obtain

$$
\left|u_{j}\right|_{k} \leq C^{\prime}
$$


Here $C^{\prime}$ is a constant independent of $j$ and we have used (4.8) in estimating $\Delta_{b(j)} u_{j}$ and dominating the $L^{2}$ norm of $u_{j}$. From (4.12) there exists a subsequence, still denoted $u_{j}$, which weakly converges to $u$ in $S_{k}$ but strongly converges to $u$ in $S_{k-1}$, say. Let $\theta=u^{2} \hat{\theta}$. Applying (4.11) to $u_{j}-u$ and using the interpolation inequality to absorb $\left|u_{j}-u\right|_{k-2}$ to the left side, we get

$$
\left|u_{j}-u\right|_{k} \lesssim\left|R_{J_{j}, \hat{\theta}}-R_{J, \hat{\theta}}\right|_{k-2}+\left|u_{j}-u\right|_{3} .
$$

Here we have used an interpolation inequality (Cor.2.11 in [BD]) to estimate $u_{j}^{3}-u^{3}$. It follows by (4.13) that $u_{j}$ tends to $u$ in $S_{k}$, and it is clear that $R_{J, \theta}=-1$ in view of (4.5),(4.6). We have proved our claim. Now consider the space $\mathfrak{J}_{-1}$ of all smooth $J$ in $\mathfrak{J}$ such that $R_{J, \theta}=-1$ for some smooth $\theta$. (unique if exists) The argument in our first paragraph shows that $\mathfrak{J}_{-1}$ is open in $\mathfrak{J}$ (in $C^{\infty}$ topology). The argument (and our claim) above shows in particular that $\mathfrak{J}_{-1}$ is closed in $C^{\infty}$ topology. Therefore $\mathfrak{J}_{-1}=\mathfrak{J}$. The lemma follows since $\mathfrak{J}^{k}$ is the completion of $\mathfrak{J}$ under the norm $|\cdot|_{k}$.

We remark that the similar idea of the above proof has been applied to the case of a fixed $\mathrm{CR}$ structure in $[\mathrm{CH}]$. We can now prove the properness of contact diffeomorphisms acting on $\mathfrak{J}$ in the case of negative pseudohermitian scalar curvature. We can talk about $S_{k}$ contact diffeomorphism on a contact manifold. (see Prop.2.18 in [BD])

Proposition 4.7. Let the assumptions be as in Lemma 4.6. Let $\phi_{j}$ be a sequence of contact diffeomorphisms in $S_{k+1}$ with $k \geq 12$. Suppose $\phi_{j}^{*} J_{j}$ and $J_{j}$ converge in $S_{k}$ as $j$ goes to infinity for $J_{j}$ in $\mathfrak{J}^{k}$. Then there exists a subsequence of $\phi_{j}$ which converges in $S_{k+1}$.

Proof. From Lemma 4.6 we can associate a unique $S_{k} \operatorname{contact}$ form $\theta_{j}$ to $J_{j}$ so that $R_{J_{j}, \theta_{j}}=-1$. Let $g_{j}$ be the adapted metric associated to $\left(J_{j}, \theta_{j}\right):([\mathrm{CH}])$ i.e. $g_{j}=\theta_{j}^{2}+d \theta_{j}\left(\cdot, J_{j}(\cdot)\right)$. $g_{j}$ converges at least in $S_{k-2}$ since $\theta_{j}$ converges in $S_{k}$ as shown in the proof of Lemma 4.6. $\phi_{j}^{*} \theta_{j}$ is just the unique contact form associated to $\widetilde{J}_{j}=\phi_{j}^{*} J_{j}$ satisfying the equation of pseudohermitian scalar curvature $=-1$. It follows that $\phi_{j}^{*} g_{j}$ converges at least in $S_{k-2} . S_{k-2}$ is contained in $H^{(k-2) / 2}$ (the usual $L^{2}$ Sobolev space) with $(k-2) / 2>4$. Therefore we can apply the result of Ebin and Palais (Theorem 2.3.1 in [Tr]) to conclude the convergence of a subsequence (still denoted $\phi_{j}$ ) of $\phi_{j}$ in $H^{k / 2}$. We need to show the convergence actually is in $S_{k+1}$. Take a smooth contact 
form $\hat{\theta}$. There is a uniquely determined smooth vector field $\hat{T}$ such that $\hat{\theta}(\hat{T})=1, d \hat{\theta}(\hat{T}, \cdot)=0$. For $\left(J_{j}, \hat{\theta}\right)$ we can choose $S_{k}$ admissible coframe $\theta_{j}^{1}$. ([Lee]) (let $e_{1}$ be a smooth local section of the contact bundle $H$. Let $\omega^{1}, \omega^{2}, \hat{\theta}$ be a local coframe dual to $e_{1}, J e_{1}, \hat{T}$. Then $\theta^{1}$ is defined to be $\omega^{1}+i \omega^{2}$ and if $J$ is in $S_{k}$, then $\omega^{1}, \omega^{2}$, hence $\theta^{1}$ is in $S_{k}$ ) Write $\theta_{j}=e^{2 g_{j}} \hat{\theta} . g_{j}$ converges in $S_{k}$ since $\theta_{j}$ converges in $S_{k}$. Also write $\phi_{j}^{*} \theta_{j}=e^{2 f_{j}} \hat{\theta} . f_{j}$ converges in $S_{k}$ since $\widetilde{J}_{j}$ converges in $S_{k}$ by the assumption. (same reasoning as in the proof of Lemma 4.6) It is easy to see

$$
\phi_{j}^{*} \hat{\theta}=e^{-2 g_{j} \circ \phi_{j}+2 f_{j}} \hat{\theta}
$$

Let $h_{j}=-g_{j} \circ \phi_{j}+f_{j}$. Let $\widetilde{\theta}_{j}^{1}$ be a local $S_{k}$ admissible coframe with respect to $\left(\widetilde{J}_{j}, \hat{\theta}\right)$. Then we can adjust $\theta_{j}^{1}$ in $S_{k}$ by a modulus 1 factor (still denoted $\left.\theta_{j}^{1}\right)$ so that

$$
\phi_{j}^{*} \theta_{j}^{1}=e^{h_{j}} \widetilde{\theta}_{j}^{1} \text { modulo } \hat{\theta} .
$$

((5.5) on p.421 in [Lee]) Now suppose $\phi_{j}$ converges in $S_{l}$ for $l \leq k$. Then $h_{j}$ converges in $S_{l}$ too. (the composition map of an $S_{l}$ function and an $S_{l}$ contact diffeomorphism is still $S_{l}$ and the map is jointly continuous for $l \geq 6$. A proof can be given by mimicking the one for the usual $L^{2}$ Sobolev spaces. See pp.15-16 in [Eb]. Also see Prop. 2.13 in [BD] for the precise estimate. Note that we start with $S_{k / 2}$ with $k / 2 \geq 6$ in which $\phi_{j}$ converges) It follows that $\phi_{j}^{*} \theta_{j}^{1}$ and $\phi_{j}^{*} \theta_{j}^{\overline{1}}$ converge in $S_{l}$ when applied to vectors tangent to the contact bundle by (4.15). Let $\widetilde{Z}_{1}=e_{1}+i \widetilde{J} e_{1}$ where $\widetilde{J}$ is the limit of $\widetilde{J}_{j}$ in $S_{k}$. Then $\phi_{j *}\left(\widetilde{Z}_{1}\right)$ and $\phi_{j *}\left(\widetilde{Z}_{\overline{1}}\right)$ converge in $S_{l}$. Therefore $\phi_{j}$ converges in $S_{\ell+1}$. Thus by induction we finally obtain that $\phi_{j}$ converges in $S_{k+1}$.

We remark that the properness of the contact action for a contact manifold is generally not true. For instance, say, $\mathfrak{J}$ contains a CR structure with noncompact $\mathrm{CR}$ automorphism group. Now we can apply Proposition 4.7 to our case $(M, H)=(\hat{S}, \hat{H})$ on which there are canonical spherical $\hat{J}$ and contact form $\hat{\theta}$ such that $R_{\hat{J}, \hat{\theta}}=-1$. (and $A_{\hat{J}, \hat{\theta}}=0$. See section 2) Let $C_{J}$ denote the group of $\mathrm{CR}$ automorphisms relative to $J$ with the identity component $C_{J}^{0}$. We have a $U(1)$ action on $\hat{S}$ given by fibre multiplications by unit-length constants. Let $\widetilde{\mathfrak{S}}^{U(1)}$ denote the space of $U(1)$ invariant elements in $\widetilde{\mathfrak{S}}$. Let $C_{\hat{H}}^{U(1), 0}$ denote the identity component of the group of $U(1)$ equivariant contact diffeomorphisms in $C_{\hat{H}}^{0}$. 
Proposition 4.8. (a) For $J$ in $\widetilde{\mathfrak{S}}^{U(1)}, C_{J}^{0}$ equals $U(1)=\{$ fibre multiplications by unit-length constants $\}$ and is contained in the center of $C_{\hat{H}}^{U(1), 0}$.

(b) $C_{\hat{H}}^{U(1), 0} / U(1)$ acts on $\widetilde{\mathfrak{S}}^{U(1)}$ freely and properly.

Proof. Any CR automorphism $\phi$ in $C_{J}^{0}$ relative to $J$ in $\widetilde{\mathfrak{S}}^{U(1)}$ is $U(1)$ equivariant by Proposition 3.14 in [Ep]. Therefore it can be pushed down to a biholomorphism on $\hat{N}$, which must be the identity since genus $(\hat{N}) \geq 2$. On the other hand $\phi$ extends to a holomorphic bundle automorphism of $\hat{L}$. Therefore $\phi$ is just a fibre multiplication by a nonzero holomorphic function on $\hat{N}$, which must be constant since $\hat{N}$ is closed (compact without boundary). (cf. Proposition 3.4) The second conclusion of (a) follows by the definition of $C_{\hat{H}}^{U(1), 0}$. Now (b) is clear by (a) and Proposition 4.7.

In the next section we will parametrize a certain open connected subspace $\mathfrak{S}_{0}^{t}$ of $\mathfrak{S}^{t, U(1)}=\widetilde{\mathfrak{S}}^{U(1)} / C_{\hat{H}}^{U(1), 0}$ as a smooth manifold and show that $\mathfrak{S}_{0}^{t}$ is diffeomorphic to $P_{i c_{0}}^{t}$, an open connected subspace of $P_{i c}^{t}$.

\section{The smooth manifold structure on $\mathfrak{S}_{0}^{t}$ : Proof of Theorem A and Corollary B.}

Let $\widetilde{P}_{i c_{0}}$ be the connected component of $\widetilde{P}_{i c}$, containing $(\hat{L}, \hat{J})$. Define $P_{i c_{0}}^{t}$ to be the quotient space of $\widetilde{P}_{i c_{0}}$ modulo the action of $\mathfrak{B}$ or Bdiff $f_{0} . P_{i c_{0}}^{t}$ is an open connected subset of $P_{i c}^{t}$. (actually they are the same since $\widetilde{P}_{i c}$ is known to be connected. But we do not pursue it here)

Given an element $(\hat{L}, \widetilde{J})$ in $\widetilde{P}_{i c_{0}}$, there associates a unique (up to a positive constant multiple) hermitian metric \|\|$_{\widetilde{J}}$ on $\hat{L}$ according to Proposition 2.1. Define $\rho: \hat{L} \backslash$ the zero section $\rightarrow \mathbb{R}$ by $\rho(s)=\|s\|_{\hat{J}} /\|s\|_{\widetilde{J}}$. Here $\hat{J}$ denotes the complex structure on $\hat{L}$ (and also $\hat{S}$ ) associated to the fixed (or reference) holomorphic line bundle $(\hat{L}, \hat{N})$ as before. It follows that $\rho(\lambda s)=\rho(s)$ for $\lambda$ in $\mathbb{C} \backslash\{0\}$, so $\rho$ can be pushed down to define a function on $\hat{N}$, still denoted $\rho$. Define $m_{\rho}: \hat{L} \rightarrow \hat{L}$ by

$$
m_{\rho}(s)=\rho(\hat{\pi}(s)) s
$$

where $\hat{\pi}: \hat{L} \rightarrow \hat{N}$ is the projection. Note that $m_{\rho}$ maps $\hat{S}=\{s \in \hat{L}:$ $\left.\|s\|_{\hat{J}}=1\right\}$ onto $S_{\widetilde{J}}=\left\{s \in \hat{L}:\|s\|_{\widetilde{J}}=1\right\}$. The contact bundle $\widetilde{H}$ defined 
by subbundle of $T \hat{S}$, invariant under the endomorphism $m_{\rho}^{*} \widetilde{J}$ restricted to $T \hat{S}$, differs from $\hat{H}$ in general. We need the $U(1)$-invariant version of Gray's theorem. Let $M$ be a closed (compact without boundary) smooth manifold of dimension $2 n+1$ with a smooth $U(1)$ action. Suppose for each $\xi$ in $U(1)$, the action $A_{\xi}$ on $M$ is a diffeomorphism. Let $\operatorname{Diff}^{U(1)}(M)$ denote the space of all $U(1)$-equivariant diffeomorphisms. Let $\mathfrak{B}^{U(1)}$ denote the space of all $U(1)$-invariant (smooth) contact bundles. It is clear that $D$ if $f^{U(1)}(M)$ acts on $\mathfrak{B}^{U(1)}$ by pushing forward. In Appendix A, we will show that both $D i f f^{U(1)}(M)$ and $\mathfrak{B}^{U(1)}$ are smooth tame Frechet manifolds in the terminology of [Ha]; we will also show the following $U(1)$-invariant version of Gray's theorem. (cf. Theorem 2.4.6 in [Ha])

Theorem 5.1. Any contact bundle near a given one $H$ in $\mathfrak{B}^{U(1)}$ is conjugate to $H$ by a U(1)-equivariant diffeomorphism near the identity. The identity component of $\operatorname{Diff}^{U(1)}(M)$ acts transitively on each component of $\mathfrak{B}^{U(1)}$.

Now apply Theorem 5.1 to our case: $M=\hat{S}$ with the $U(1)$ action given by fibre multiplications by unit-length constants. (cf. section 4) Since $m_{\rho}$ is $U(1)$-equivariant $(U(1)$ action also defined on $\hat{L}), \widetilde{H}$ is $U(1)$-invariant, so there exists a $U(1)$-equivariant diffeomorphism $\phi$ with $\phi_{*} \hat{H}=\widetilde{H}$. Note that two choices of such $\phi$ are different by $U(1)$-equivariant contact diffeomorphisms, i.e. the inverse of the one composed with the other belongs to $C_{\hat{H}}^{U(1), 0}$. Using $\phi$ to pull back the $U(1)$-invariant CR structure $\left(\widetilde{H}, m_{\rho}^{*} \widetilde{J} \mid \widetilde{H}\right)$ on $\hat{S}$, we obtain a $U(1)$-invariant CR structure $J=\left(m_{\rho} \circ \phi\right)^{*}(\widetilde{J}) \mid \hat{H}$ in $\widetilde{\mathfrak{S}}^{U(1)}$. Define $\widetilde{\tau}: \widetilde{P}_{i c_{0}} \rightarrow \widetilde{\mathfrak{S}}^{U(1)}$ by $\widetilde{\tau}(\hat{L}, \widetilde{J})=(\hat{S}, \hat{H}, J)$ where $J=\left(m_{\rho} \circ \phi\right)^{*}(\widetilde{J}) \mid \hat{H}$. The map $\tilde{\tau}$ gives rise to a map

$$
\tau^{t}: P_{i c_{0}}^{t} \rightarrow \mathfrak{S}^{t, U(1)}
$$

("uniqueness" of \|\|$_{\tilde{J}}$ by Proposition 2.1) Recall that

$$
\mathfrak{S}^{t, U(1)}=\widetilde{\mathfrak{S}}^{U(1)} / C_{\hat{H}}^{U(1), 0} .
$$

Endow $\widetilde{\mathfrak{S}}^{U(1)}$ with the $C^{\infty}$-topology so that $\mathfrak{S}^{t, U(1)}$ has the induced quotient topology.

Proposition 5.2. The map $\tau^{t}: P_{i c_{0}}^{t} \rightarrow \mathfrak{S}^{t, U(1)}$ is a homeomorphism onto its image. 
Proof. To prove $\tau^{t}$ is continuous, we will suitably choose a unique \|\|$_{\widetilde{J}}$ and a unique $\phi$ for a given $\widetilde{J}$. Remember \|\|$_{\widetilde{J}}$ is determined by $\lambda$ in (2.3). We normalize the solution $\lambda$ of (2.3) by requiring $\lambda=1$ at some point $\mathrm{p}$, so $\lambda$ is uniquely determined. Furthermore, the map : $\widetilde{J} \rightarrow \lambda$ is continuous by the standard arguments in the elliptic theory. (apply the Harnack estimates (Theorems 9.20, 9.22 in [GT]) to get upper bounds for $\log \lambda$ and $\log \lambda^{-1}$ (cf.(3.22))) Let Diff $f_{0}^{U(1)}(M)$ denote the identity component of $\operatorname{Dif} f^{U(1)}(M)$. Let $\mathfrak{B}_{0}^{U(1)}$ denote the connected component of $\mathfrak{B}^{U(1)}$, containing $\hat{H}$. To pick up a unique $\phi$, we invoke the following result.

Lemma 5.3. There is a local smooth tame map $s: \mathfrak{B}_{0}^{U(1)} \rightarrow D$ iff $f_{0}^{U(1)}(M)$ near a reference point $H_{0}$ such that $s(\widetilde{H})_{*}\left(H_{0}\right)=\widetilde{H}$.

We will prove Lemma 5.3 in Appendix A. By Lemma 5.3, the map: $\widetilde{J} \rightarrow$ $\widetilde{H}$ composed with $s$ gives a continuous map: $\widetilde{J} \rightarrow \phi$ near a reference point. We have shown that $\tau^{t}$ is continuous. On the other hand, given $J$ in $\widetilde{\mathfrak{S}}^{U(1)}$, we can extend $J$ to $\widetilde{J}$ in $\widetilde{P}_{i c}$ as below. For $y$ not in the 0 -section of $\hat{L}$, let $\rho=\|y\|_{\hat{J}}$ and define $\widetilde{J}_{y}$ by the fibre dilation: $\widetilde{J}_{y}(v)=J_{x}\left(m_{\rho *}^{-1}(v)\right)$ for $v$ in $m_{\rho *} \hat{H}_{x}, x=m_{\rho}^{-1}(y)$. Since $J$ is $U(1)$-invariant, it can be pushed down to define a complex structure $c$ on $\hat{N}: c\left(\hat{\pi}_{*}(v)\right)=\hat{\pi}_{*}(J v)$ for $v$ in $\hat{H}_{\ell}, \ell$ in $\hat{S}$. Here we identify $\hat{H}_{\ell}$ with the tangent space of $\hat{N}$ at $\hat{\pi}(\ell)$. Let $s_{0}$ denote the 0 -section: $\hat{N} \rightarrow \hat{L}$. For $y$ in $s_{0}(\hat{N})$, we define $\widetilde{J}_{y}(v)=s_{0 *} c\left(\hat{\pi}_{*}(v)\right)$ for $v$ in $T_{y}\left(s_{0}(\hat{N})\right)$. For $v$ tangent to fibres, we just define $\widetilde{J}$ to be the usual complex structure on $\mathbb{C}$ in local trivializations. Now it is a matter to verify that $\widetilde{J}$ is smooth and hence belongs to $\widetilde{P}_{i c}$. First observe that the 2-plane distribution $\mathfrak{D}$ on $\hat{L}$ defined by $m_{\rho *} \hat{H}(\rho \in \mathbb{C} \backslash\{0\})$ and tangent spaces of $s_{0} \hat{N}$ is smooth. (in a local trivialization $(z, w)$, this distribution can be described by the kernel of the one-form $i h_{z} w d z+i h d w$. Here $h(z, \bar{z})=\|s(z)\|_{\hat{J}}^{2}$ for a local holomorphic section s. cf. (2.4)) To show $\widetilde{J}$ is smooth, it is enough to prove $\widetilde{J}(v)$ is smooth for every smooth vector field $v$. Write $v=v_{\mathfrak{D}}+v_{f}$. Here $v_{\mathfrak{D}}$ in $\mathfrak{D}$ is smooth while $v_{f}$ is a smooth vector field tangent to the fibres. It is obvious that $\widetilde{J}\left(v_{f}\right)$ is smooth. Let $i_{l}$ denote the linear isomorphism: $T_{\hat{\pi}(\ell)} \hat{N} \rightarrow \mathfrak{D}_{\ell}$ for $\ell \in \hat{L}$ so that $i_{\ell} \circ \hat{\pi}_{*}=$ identity on $\mathfrak{D}_{\ell}$. Note that $i_{\ell}=s_{0 *}$ at $\hat{\pi}(\ell)$ for $\ell$ in $s_{0}(\hat{N})$. Now we can express

$$
\widetilde{J}_{\ell}\left(v_{\mathfrak{D}}(\ell)\right)=i_{\ell} \circ c\left(\hat{\pi}_{*}\left(v_{\mathfrak{D}}(\ell)\right)\right) .
$$

Since $i: \ell \rightarrow i_{\ell}$ is smooth as viewed as a section of $\operatorname{End}\left(\hat{\pi}^{*}(T \hat{N}), \mathfrak{D}\right)$ over $\hat{L}$, it follows that $\widetilde{J}\left(v_{\mathfrak{D}}\right)$ is smooth, hence $\widetilde{J}$ is smooth. It is not hard to see 
that the map $\widetilde{\text { ext }}: \widetilde{\mathfrak{S}}^{U(1)} \rightarrow \widetilde{P}_{i c}$ defined by $\widetilde{\operatorname{ext}}(J)=\widetilde{J}$ is continuous. (see also [Ep] for precise construction in a local trivialization and in terms of type $(0,1)$ vector fields) Moreover, $\widetilde{e x t}$ induces a continuous map ext from $\mathfrak{S}^{t, U(1)}$ to $P_{i c}^{t}$ by the proof of Proposition 2.4 and $e x t \circ \tau^{t}$ equals the identity for the same reason. Therefore $\tau^{t}$ is injective and hence a homeomorphism onto its image.

In fact $\tau^{t}$ is surjective onto the connected component of $\mathfrak{S}^{t, U(1)}$, containing the reference element $[\hat{J}]$. We will see this below. First let us determine the universal cover of $(\hat{S}, \hat{H}, \hat{J})$. Denote the unit disc in the complex plane $C$ by $D$. Define the hermitian metric \|\|$_{e}$ on the trivial holomorphic line bundle $D \times C$ by

$$
\|(z, w)\|_{e}=|w|^{2} /\left(1-|z|^{2}\right)^{e}
$$

for $e=m /(g-1)$. (recall that $-m$ is the first Chern number of $\hat{L}$ and $g$ is the genus of $\hat{N})$ It is a direct verification that $h(z, \bar{z})=\|(z, 1)\|_{e}$ satisfies (2.1) in $D$, the universal cover of $\hat{N}$. Write an element $A$ in $U(1,1) \times U(1)$ as below:

$$
A=\left(\begin{array}{lll}
a & b & 0 \\
c & d & 0 \\
0 & 0 & u
\end{array}\right)
$$

for $u$ in $U(1),\left(\begin{array}{ll}a & b \\ c & d\end{array}\right)$ in $U(1,1)$ with respect to the quadratic form given by $\left(\begin{array}{cc}1 & 0 \\ 0 & -1\end{array}\right)$. The group $U(1,1) \times U(1)$ acts on $D \times C$ by

$$
A(z, w)=\left((a z+b) /(c z+d), u w /(c z+d)^{e}\right) .
$$

It is easy to see that $A$ leaves \|\|$_{e}$ invariant. (just note that $|z|^{2}-|w|^{2}=$ $\left.|a z+b w|^{2}-|c z+d w|^{2}\right)$ Define $S_{e} \subset D \times C$ by \|\|$_{e}=1$. It follows that $S_{1 /(g-1)}$ is an $m$ to 1 cover of $S_{m /(g-1)}=S_{e}$ and a $g-1$ to 1 cover of $S_{1}$. Since $S_{1}(=$ $\left.S^{3} \backslash\{w=0\}\right)$ is obviously spherical, $S_{e}$ is spherical too. The holomorphic line bundle $\hat{L}$ over the Riemann surface $\hat{N}$ gives rise to a representation of $\pi_{1}(\hat{N})$ in $P U(1,1) \times U(1)$ acting on $D \times C$. Here $P U(1,1)=S U(1,1) /$ center acts on $D$ as holomorphic transformations. It follows that $S_{e} / \pi_{1}(\hat{N})$ is a spherical circle bundle of $\hat{L}$ over $\hat{N}$ with the hermitian metric induced from \|\|$_{e}$ satisfying (2.1). By uniqueness (up to a constant multiple) $\hat{S}=S_{e} / \pi_{1}(\hat{N})$. As a consequence, the universal cover of $\hat{S}$ (as CR manifold), denoted $\widehat{S}$, is the same for any $\left(g, c_{1}\right)$ and is the infinite cyclic cover of $S_{1}=S^{3} \backslash\{w=0\}$. It is well known (e.g. [BS]) that $\widetilde{S}$ is homogeneous. 
Proposition 5.4. Every element in $\widetilde{\mathfrak{S}}^{0}$ is $U(1)$-invariant up to a contact diffeomorphism in $C_{\hat{H}}^{0}$.

Proof. First note that every spherical CR manifold is locally homogeneous in the weak sense (i.e. any two points have isomorphic neighborhoods). By Theorem 8.2 of [ENS] or Theorem 4 of [Go], the universal cover of any element in $\widetilde{\mathfrak{S}}^{0}$ is homogeneous and hence is CR equivalent to $\widetilde{S}$ by the classification. ([BS] or [ENS]) Denote $\Gamma$ the fundamental group of $\hat{S}$. It is not hard to see (e.g. [FG] p.44) by the theorem of Seifert and Van Kampen that $\Gamma$ has a presentation:

$$
\Gamma=\left\langle a_{1}, b_{1}, \ldots, a_{g}, b_{g}, h: \prod_{i=1}^{i=g}\left[a_{i}, b_{i}\right]=h^{-m}, h \text { central }\right\rangle
$$

$(-m$ is the first Chern number or Euler number). Realize $\Gamma$ as Deck transformations of $\widetilde{S}$ via the homomorphism $j: \Gamma \rightarrow \operatorname{Aut}_{C R}(\widetilde{S})$. It is known ([BS], p.234) that $\operatorname{Aut}_{C R}(\widetilde{S})$ satisfies the following exact sequence:

$$
0 \longrightarrow \mathbb{R} \longrightarrow \operatorname{Aut}_{C R}(\widetilde{S}) \stackrel{p r}{\longrightarrow} P U(1,1) \longrightarrow 1 .
$$

We claim that $p r \circ j(h)=I$, the identity. Since the quotient space $j(\Gamma) \backslash \widetilde{S}$ is CR equivalent to $(\hat{S}, \hat{H}, J)$ for some $J$ in $\widetilde{\mathfrak{S}}^{0}$, it is compact, and hence has finite invariant measure. $\operatorname{Aut}_{C R}(\widetilde{S})$ acts on $\widetilde{S}$ transitively with the compact isotropy group, isomorphic to $U(1)$, so $j(\Gamma) \backslash \operatorname{Aut}_{C R}(\widetilde{S})$ is compact, and hence has finite invariant measure. Let $H=p r \circ j(\Gamma)$. It follows that $H \backslash P U(1,1)$ is compact and has finite invariant measure. By Lemma 5.4 in [Ra], $\mathrm{H}$ has property $(\mathrm{S})$ in $P U(1,1)$. Therefore by Corollary 5.18 in [Ra] the centralizer $Z(H)$ of $H$ in $P U(1,1)$ is the centre of $P U(1,1)$, which consists of the identity. Now note that $\operatorname{pr} \circ j(h)$ is in $Z(H)$ since h is central. Hence $\operatorname{pr} \circ j(h)=I$, so $h$ is mapped into the $\mathbb{R}$ part by $j$. Let dev denote the developing map from $\widetilde{S}$ onto $S_{1} \subset S^{3}$. Let hol denote the holonomy map from $\operatorname{Aut}_{C R}(\widetilde{S})$ onto $\operatorname{Aut}_{C R}\left(S_{1}\right)=P U(1,1) \times U(1) \subset \operatorname{Aut}_{C R}\left(S^{3}\right)=$ $P U(2,1)$. The developing pair (hol, dev) induces naturally another pair $\left(h o l^{\prime}, \operatorname{dev}^{\prime}\right):\left(\operatorname{Aut}_{C R}(\widetilde{S}), \widetilde{S}\right) \rightarrow\left(\operatorname{Aut}_{C R}\left(S_{e}\right), S_{e}\right)$ by noting that both $S_{1}$ and $S_{e}$ are covered by the common covering space $S_{1 /(g-1)}$. Let $a_{i}^{\prime}, b_{i}^{\prime}, h^{\prime}$ denote the corresponding generators of $a_{i}, b_{i}, h$ under the map $h o l^{\prime} \circ j$, respectively. By projecting the commutator relation in $\Gamma$ into the $U(1)$ part of $\operatorname{Aut}_{C R}\left(S_{e}\right)$, we obtain $I=\left(h^{\prime}\right)^{-m}$. But for $\hat{J}, h^{\prime}=I$. Hence for $J$ in $\widetilde{\mathfrak{S}}^{0}, h^{\prime}$ is also equal to the identity by continuity. (note that the representation map $j$ depends on our spherical CR structure $J$ on $(\hat{S}, \hat{H}))$ Thus the subgroup hol' $\circ j(\Gamma)$ of $\operatorname{Aut}_{C R}\left(S_{e}\right)$ can be viewed as a representation of $\pi_{1}(\hat{N})$ generated by 
$a_{i}^{\prime}, b_{i}^{\prime}$ in $\operatorname{Aut}_{C R}\left(S_{e}\right)$. Therefore $h o l^{\prime} \circ j(\Gamma) \backslash S_{e}$ is the spherical circle bundle $S_{L}$ of some holomorphic line bundle $L$, determined by Proposition 2.1, as discussed previously. (in particular it is $U(1)$-invariant) On the other hand, $h o l^{\prime} \circ j(\Gamma) \backslash S_{e}$ is CR equivalent to $j(\Gamma) \backslash \widetilde{S}$ representing $(\hat{S}, \hat{H}, J)$ in view of $d e v^{\prime}$ being a covering map. Let $\Sigma$ denote the CR isomorphism from $(\hat{S}, \hat{H}, J)$ onto $S_{L}$. Composing a bundle isomorphism between $\hat{L}$ and $L$ with a fibre multiplication map $m_{\rho}$, we can construct a $U(1)$-equivariant diffeomorphism $\phi: \hat{S} \rightarrow S_{L}$. (note that $\Sigma$ may not be $U(1)$-equivariant) Now $\phi^{*} H_{L}$ and $\phi^{*} J_{L}$ are invariant with respect to the $U(1)$-action on $\hat{S}$. By Theorem 5.1 we can find a $U(1)$-equivariant diffeomorphism $\psi$ such that $\psi \circ \phi^{-1} \circ \Sigma$ is in $C_{\hat{H}}^{0}$ while $\left(\psi \circ \phi^{-1} \circ \Sigma\right)^{-1 *}(J)=\psi^{-1 *} \circ \phi^{*}\left(J_{L}\right)$ is $U(1)$-invariant.

Let $\widetilde{\mathfrak{S}}^{0}$ denote the connected component of $\widetilde{\mathfrak{S}}$, containing $(\hat{S}, \hat{H}, \hat{J})$. Let $\widetilde{\mathfrak{S}}^{0, U(1)}$ denote the space of $U(1)$-invariant elements in $\widetilde{\mathfrak{S}}^{0}$. Any $(\hat{S}, \hat{H}, J)$ in $\widetilde{\mathfrak{S}}^{0, U(1)}$ extends to a complex structure $\widetilde{J}$ on $\hat{L}$. The argument in the above proof of Proposition 5.4 shows that $(\hat{S}, \hat{H}, J)$ is CR-equivalent to $S_{L}$ for a certain holomorphic line bundle $L$. The CR isomorphism between $(\hat{S}, \hat{H}, J)$ and $S_{L}$ implies the existence of a holomorphic bundle isomorphism between $(\hat{L}, \widetilde{J})$ and $L$ in view of the proof of Proposition 2.4. Since $S_{L}$ is uniquely determined by $L$ (Proposition 2.1), it follows that $(\hat{S}, \hat{H}, J)$ is uniquely determined by $(\hat{L}, \widetilde{J})$, i.e. suppose two $\left(\hat{S}, \hat{H}, J_{i}\right)$ have isomorphic extensions $\left(\hat{L}, \widetilde{J}_{i}\right), i=1,2$, then $\left(\hat{S}, \hat{H}, J_{1}\right)$ is CR-equivalent to $\left(\hat{S}, \hat{H}, J_{2}\right)$. Furthermore by Proposition 3.14 in [Ep] we have

Lemma 5.5. Let $\left(\hat{L}, \widetilde{J}_{i}\right)$ be the extension of $\left(\hat{S}, \hat{H}, J_{i}\right)$ in $\widetilde{\mathfrak{S}}^{0, U(1)}, i=1,2$. Suppose $\left(\hat{L}, \widetilde{J}_{1}\right)$ is isomorphic to $\left(\hat{L}, \widetilde{J}_{2}\right)$ by a bundle automorphism in Bdiff $f_{0}$. Then $\left(\hat{S}, \hat{H}, J_{1}\right)$ is CR-equivalent to $\left(\hat{S}, \hat{H}, J_{2}\right)$ by a contact diffeomorphism in $C_{\hat{H}}^{U(1), 0}$.

Let $\widetilde{\mathfrak{S}}^{U(1), 0}$ denote the connected component of $\widetilde{\mathfrak{S}}^{U(1)}$, containing $(\hat{S}, \hat{H}, \hat{J})$. Define $\mathfrak{S}_{0}^{t}$ to be the quotient space of $\widetilde{\mathfrak{S}}^{U(1), 0}$ modulo $C_{\hat{H}}^{U(1), 0}$ (or $C_{\hat{H}}^{0}$ : two quotient spaces are the same by the above discussion), i.e. two elements in $\widetilde{\mathfrak{S}}^{U(1), 0}$ are equivalent if one is carried to another by a contact diffeomorphism in $C_{\hat{H}}^{U(1), 0}$ (or $C_{\hat{H}}^{0}$ resp.) by pulling back. Observe that $\mathfrak{S}_{0}^{t}$ is an open connected subset of $\widetilde{\mathfrak{S}}^{0, U(1)} / C_{\hat{H}}^{0}$ which equals $\widetilde{\mathfrak{S}}^{0} / C_{\hat{H}}^{0}$ in view of Proposition 5.4. Since a $\mathrm{CR}$ equivalence $\phi$ between two $U(1)$-invariant $\mathrm{CR}$ circle bundles is $U(1)$-equivariant, we have $\mathfrak{S}^{t, U(1)}\left(=\widetilde{\mathfrak{S}}^{U(1)} / C_{\hat{H}}^{U(1), 0}\right)=\widetilde{\mathfrak{S}}^{U(1)} / C_{\hat{H}}^{0}$.

Proposition 5.6. $\tau^{t}: P_{i c_{0}}^{t} \rightarrow \mathfrak{S}_{0}^{t}$ is surjective and a homeomorphism in 
view of Proposition 5.2.

Proof. Given an element $(\hat{S}, \hat{H}, J)$ in $\widetilde{\mathfrak{S}}^{U(1), 0}$, there associates an extension $(\hat{L}, \widetilde{J})$ in $\widetilde{P}_{i c_{0}}$. We claim $\tau^{t}([(\hat{L}, \widetilde{J})])=[(\hat{S}, \hat{H}, J)]$. Recall that the construction of $\tau^{t}$ involves a map $m_{\rho}$ and a $U(1)$-equivariant diffeomorphism $\phi$ on $\hat{S}$. Extend $\phi$ to a bundle automorphism $\widetilde{\phi}$ in Bdiff $0 . \tilde{\tau}(\hat{L}, \widetilde{J})=$ $\left(\hat{S}, \hat{H},\left(m_{\rho} \circ \phi\right)^{*}(\hat{J}) \mid \hat{H}\right)$ is the restriction of $\left(m_{\rho} \circ \widetilde{\phi}\right)^{*}(\widetilde{J})$ on $\hat{L}$ to $(\hat{S}, \hat{H})$. Since $m_{\rho} \circ \tilde{\phi}$ is a bundle automorphism of $\hat{L}$ in Bdiff $f_{0}$, it follows that $\left(\hat{S}, \hat{H},\left(m_{\rho} \circ \phi\right)^{*}(\widetilde{J})\right)$ is CR-equivalent to $(\hat{S}, \hat{H}, J)$ by a contact diffeomorphism in $C_{\hat{H}}^{U(1), 0}$ according to Lemma 5.5 .

We remark that in $[\mathrm{KT}]$ Kamishima and Tan studied the deformation space of $U(1)$-invariant spherical CR-structures by analyzing the space of developing pairs. Their deformation space for $M=\hat{S}$ is in one-to-one correspondence with our space $\mathfrak{S}^{t, U(1)}$ by "contact" reduction. According to Corollary 5.2.2 in [KT], this space is homeomorphic to $\operatorname{Hom}\left(\pi_{1}(\hat{N}), P U(1,1)\right) / P U(1,1) \times T^{2 g}$, and it is well known that the dimension of $\operatorname{Hom}\left(\pi_{1}(\hat{N}), P U(1,1)\right) / P U(1,1)$ is $6 g-6$, the dimension of Teichmuller space.(e.g. [Go]) Thus the total dimension is $6 g-6+2 g=8 g-6$ (cf. Theorem 6 (d) in [Go]) while Proposition 5.6 shows that an open connected subset $\mathfrak{S}_{0}^{t}$ of $\mathfrak{S}^{t, U(1)}$ is homeomorphic to $P_{i c_{0}}^{t}$ of the same dimension by Theorem 3.9.

Next we want to endow $\mathfrak{S}_{0}^{t}$ with a natural differentiable structure through the general local slice theorem, and with this differentiable structure on $\mathfrak{S}_{0}^{t}, \tau^{t}$ in Proposition 5.6 is a diffeomorphism. Given $J$ in $\widetilde{\mathfrak{S}}^{U(1), 0}$, there passes a local slice $\mathfrak{S}$ of $\mathfrak{J}$ according to Theorem A of [CL2]. Let $\mathfrak{P}$ denote the diffeomorphism given in Theorem A (1) of [CL2]. Define $\psi: \widetilde{P}_{i c_{0}} \rightarrow \mathfrak{S}$ near $(\hat{L}, \widetilde{J})$ with $\widetilde{\tau}(\hat{L}, \widetilde{J})=(\hat{S}, \hat{H}, J)$ by

$$
\psi=\operatorname{proj}_{S} \circ \mathfrak{P}^{-1} \circ \tilde{\tau} \text {. }
$$

Here $\operatorname{proj}_{S}$ denotes the projection onto the $\mathfrak{S}$-component. Since the pullback by a contact diffeomorphism does not change the vanishing of the Cartan tensor, $\psi$ actually maps into $\mathfrak{Q}^{-1}(0)$. At $(\hat{L}, \widetilde{J})$, there passes a local slice, denoted $\mathfrak{S}_{P i c}$, by Lemma 3.7. We claim $\psi: \mathfrak{S}_{P i c} \rightarrow \mathfrak{Q}^{-1}(0)$ is an immersion (between two finite dimensional manifolds) by choosing unique $\rho$ and $\phi$ in defining $\tau$ as explained in the proof of Proposition 5.2. First note that the action of bundle automorphisms does not change the transversality of tangent vectors at $\widetilde{J}$ in $\widetilde{P}_{i c_{0}}$. (here transversality means transverse to the 
orbit of $\mathfrak{B}$ or $B$ dif $f_{0}$ acting on $\widetilde{J}$ ) Use the bundle automorphism $m_{\rho} \circ \widetilde{\phi}$ in the proof of Proposition 5.6 to reduce our immersion problem to the following:

Lemma 5.7. Let $J$ in $\widetilde{\mathfrak{S}}^{U(1), 0}$ be the restriction of its extension $\widetilde{J}$ in $\widetilde{P}_{i c_{0}}$. Let $\widetilde{J}^{\prime}$ be an infinitesimal variation of $\widetilde{J}$ and $J^{\prime}$ be the corresponding infinitesimal variation of $J$ in $\mathfrak{J}$. Suppose $J^{\prime}$ is tangent to the orbit of $C_{\hat{H}}^{0}$ acting on $J$. Then $\widetilde{J}^{\prime}$ is also tangent to the orbit of $\mathfrak{B}$ acting on $\widetilde{J}$.

Proof. Take a local trivialization $(z, w)$ of $\hat{L}$ relative to $\widetilde{J}$ with $w$ being the fibre coordinate. Let $s$ be the local holomorphic section given by $z \rightarrow(z, 1)$. Let $h=h(z, \bar{z})=\|s(z)\|^{2}$ where the hermitian metric \|\| is chosen according to Proposition 2.1. By Lemma $5.5 \hat{S}$ is precisely discribed by \|\|$=1$ or $h w \bar{w}=1$ in the above local trivialization. It is easy to verify that $Z=$ $\partial_{z}-(\log h)_{z} w \partial_{w}$ is tangent to $\hat{S}$. Let $\theta^{1}=d z, \theta^{2}=d w+(\log h)_{z} w d z$. Then $\left\{\theta^{1}, \theta^{2}\right\}$ is dual to $\left\{Z, \partial_{w}\right\}$. Now we can write $J=i \theta^{1} \otimes Z+$ conjugate and $\widetilde{J}=J+\left(i \theta^{2} \otimes \partial_{w}+\right.$ conjugate $)$. Moreover let $\widetilde{J}_{t}$ be a family of extensions of $J_{t}$ with $\widetilde{J}_{0}=\widetilde{J}, J_{0}=J$. Let $Z_{t}=Z+a_{t} \bar{Z}$ be a frame of type $(1,0)$ with respect to $J_{t}$ with $a_{0}=0$. (i.e. an eigenvector of $J_{t}$ with eigenvalue i) Let $\theta_{t}^{1}=\left(\theta^{1}-\left(\bar{a}_{t}\right) \theta^{\overline{1}}\right) /\left(1-\left|a_{t}\right|^{2}\right)$. It is straightforward to determine $\theta_{t}^{2}$ such that $\left\{\theta_{t}^{1}, \theta_{t}^{2}, \theta_{t}^{1}, \theta_{t}^{2}\right\}$ is dual to $\left\{Z_{t}, \partial_{w}, \bar{Z}_{t}, \partial_{\bar{w}}\right\}$. The result is $\theta_{t}^{2}=$ $d w+(\log h)_{z} w \theta_{t}^{1}+\bar{a}_{t}(\log h)_{z} w \theta_{t}^{\overline{1}}$.

It follows that $\widetilde{J}_{t}=J_{t}+\left(i \theta_{t}^{2} \otimes \partial_{w}+\right.$ conjugate $)$. Computing the derivative at $t=0$ gives

$$
\widetilde{J}_{t}^{\prime}=J_{t}^{\prime}=2 i a_{t}^{\prime} d z \otimes \bar{Z}+\text { conjugate }
$$

by observing that $\theta_{t}^{2^{\prime}}=0$. Writing $\widetilde{J}_{t}^{\prime}=E_{1}{ }^{\overline{1}} d z \otimes \partial_{\bar{z}}+E_{1}{ }^{*} \bar{w} d z \otimes \partial_{\bar{w}}+$ conjugate (cf. (3.6)), we obtain $E_{1}{ }^{\overline{1}}=2 i a_{t}^{\prime}, E_{1}{ }^{*}=-2 i a_{t}^{\prime}(\log h)_{\bar{z}}$. Hence $E_{1}=E_{1}{ }^{*}+E_{1}{ }^{\overline{1}} \bar{\Gamma}=0$ (cf.(3.24)) by noting that $\Gamma=(\log h)_{z}$. Now by the assumption we can write $J^{\prime}=B_{J}^{\prime}(f)=f_{, 1}{ }^{1} \theta^{1} \otimes \bar{Z}+$ conjugate. (in [CL1] we write $Z_{1}$ instead of $Z$, and choosing the specific contact form (2.4), we have the torsion $A_{1}{ }^{\overline{1}}$ to vanish by (2.6)) That is to say, $2 i a_{t}^{\prime}=f_{, 1}{ }^{\overline{1}}$. To show $\widetilde{J}^{\prime}=$ $P(V)$ for some $V$ represented by $\left(v^{1}, v\right)$ (cf. (3.31)), we take $v^{1}=f,{ }^{1} / 2 i$ and $v=-\mu f / 2 i$. It follows that $P(V)=2 i\left(v^{1}{ }_{, 1}, v_{, \overline{1}}+\mu v_{\overline{1}}\right)=\left(f,{ }_{\overline{1}}, 0\right)=\left(E_{\overline{1}}{ }^{1}, 0\right)$ which represents $\widetilde{J^{\prime}}$.

Proof of Theorem A. By Lemma 5.7 the differential of $\widetilde{\tau}$ maps a tangent vector of $\mathfrak{S}_{P i c}$ at $\widetilde{J}$ to a tangent vector transverse to the orbit of $C_{\hat{H}}^{0}$ acting on $J$. It follows that the differential of $\psi$ (cf.(5.1)): $\mathfrak{S}_{\text {Pic }} \rightarrow \mathfrak{Q}^{-1}(0)$ is 
injective. Therefore $\psi \mid \mathfrak{S}_{P i c}$ is an immersion by the inverse function theorem, so $\psi \mid \mathfrak{S}_{P_{i c}}$ gives rise to a local coordinate map for $\mathfrak{S}_{0}^{t}$ near $[\mathrm{J}]$ in view of Proposition 5.6. Transition functions of these coordinate maps are smooth because $\mathfrak{S}_{\text {Pic }}$ 's can be viewed as local coordinate neighborhoods for the smooth manifold $P_{i c}^{t}$. (cf. Theorem C) Thus $\mathfrak{S}_{0}^{t}$ is a smooth manifold and in the way to define its differentiable structure we actually obtain that $\tau^{t}$ is a diffeomorphism. Hence the dimension of $\mathfrak{S}_{0}^{t}$ equals the dimension of $P_{i c}^{t}$, which is $2(4 g-3)=8 g-6$ by Theorem C.

Proof of Corollary B. $\iota$ is well defined in view of Proposition 5.4. ¿From the proof of Proposition 5.4 we learn that any spherical $(\hat{S}, \hat{H}, J)$ in $\widetilde{\mathfrak{S}}^{0}$ is CRequivalent to $S_{L}$ for some holomorphic line bundle L. Let $\phi:(\hat{S}, \hat{H}, J) \rightarrow$ $\left(S_{L}, H_{L}, J_{L}\right)$ denote this CR-isomorphism. Take $\theta=\phi^{*}\left(\theta_{L}\right)$. It is obvious that $(\hat{S}, \hat{H}, J, \theta)$ is in $\widetilde{\mathfrak{M}}_{-1,0}^{0}$ and $\iota$ maps $[(\hat{S}, \hat{H}, J, \theta)]$ to $[(\hat{S}, \hat{H}, J)]$. Thus $\iota$ is surjective and hence bijective in view of Corollary 2.3. On the other hand it is easy to see that both $\iota$ and its inverse are continuous, so $\iota$ is a homeomorphism.

\section{Appendix A: The $U(1)$-invariant version of Gray's theorem.}

We will prove Theorem 5.1 and Lemma 5.3. First denote the smooth $U(1)$ action by $U_{\rho}, 0 \leq \rho \leq 2 \pi$ with $U_{0}=U_{2 \pi}$. Pick a $U(1)$-invariant metric $g$. (which can be obtained by averaging the action on an arbitrary metric) Any $U(1)$-invariant contact bundle $H$ in $\mathfrak{B}^{U(1)}$ can be uniquely determined by a $U$ (1)-invariant 1-form $\theta$ with $\theta \wedge d \theta \neq 0$ and $|\theta|_{g}=1$. Here ||$_{g}$ denotes the pointwise length with respect to the metric $g$. Still denote the space of all such 1 -forms by $\mathfrak{B}^{U(1)}$.

Lemma A.1. $\mathfrak{B}^{U(1)}$ is a tame Frechet submanifold of the tame Frechet manifold $\mathfrak{B}$.

Proof. Let $\Omega^{1}\left(\Omega_{U(1)}^{1}\right.$, respectively) denote the space of all smooth $(U(1)$ invariant, respectively) 1 -forms on our closed manifold $M$. It is known that $\Omega^{1}$ is a tame Frechet space ([Ha]). Since the process of averaging the $U(1)$ action on a 1 -form is a tame linear map from $\Omega^{1}$ to $\Omega_{U(1)}^{1}$, it follows that $\Omega_{U(1)}^{1}$ is a tame direct summand, hence a tame Frechet space. (Lemma 1.3.3 on p.136 in [Ha]) Now consider the space

$$
T_{\theta} \mathfrak{B}^{U(1)}:=\left\{\text { smooth } 1 \text {-form } \eta:\langle\eta, \theta\rangle_{g}=0, U_{\rho}^{*} \eta=\eta\right\}
$$


where $\langle,\rangle_{g}$ denote the pointwise inner product with respect to $g$. It is easy to see that the linear map proj $: \Omega_{U(1)}^{1} \rightarrow T_{\theta} \mathfrak{B}^{U(1)}$ given by $\operatorname{proj}(\eta)=$ $\eta-\langle\eta, \theta\rangle_{g} \theta$ is tame. Therefore $T_{\theta} \mathfrak{B}^{U(1)}$ is a tame direct summand of $\Omega_{U(1)}^{1}$, hence a tame Frechet space. Define a map $\Phi_{\theta}: T_{\theta} \mathfrak{B}^{U(1)} \rightarrow \mathfrak{B}^{U(1)}$ by

$$
\Phi_{\theta}(\eta)=(\eta+\theta) /|\eta+\theta|_{g} .
$$

If we endow $\mathfrak{B}^{U(1)}$ with the $C^{\infty}$ topology, then $\Phi_{\theta}$ is a local homeomorphism near 0 with its inverse $\pi_{\theta}$ given by

$$
\pi_{\theta}(\eta)=\eta /\langle\eta, \theta\rangle_{g}-\theta
$$

Now we can compute the transition function for the overlap of two neighborhoods centered at $\theta$ and $\theta^{\prime}$ :

$$
\pi_{\theta^{\prime}} \circ \Phi_{\theta}(\eta)=(\eta+\theta) /\left\langle\eta+\theta, \theta^{\prime}\right\rangle_{g}-\theta^{\prime} .
$$

It is easy to see that $\pi_{\theta^{\prime}} \circ \Phi_{\theta}$ is smooth tame. We have shown that $\mathfrak{B}^{U(1)}$ is a tame Frechet manifold. Actually the map $\Phi_{\theta}$ also parametrizes $\mathfrak{B}$ near $\theta$. Therefore $\mathfrak{B}^{U(1)}$ is a tame Frechet submanifold of $\mathfrak{B}$.

Lemma A.2. $\operatorname{Diff}^{U(1)}(M)$ is a tame Frechet submanifold of Diff $(M)$, the group of smooth diffeomorphisms on $M$. Moreover, Diff ${ }^{U(1)}(M)$ is a smooth tame Lie group.

Proof. Let $T_{e} \operatorname{Diff}(M)\left(T_{e} D i f f^{U(1)}(M)\right.$, respectively) denote the space of all smooth (U(1)-invariant, respectively) vector fields on $M$. Here $e$ denotes the identity diffeomorphism. It is easy to see that the map pr: $T_{e} \operatorname{Diff}(M) \rightarrow$ $T_{e} \operatorname{Diff}^{U(1)}(M)$ given by

$$
\operatorname{pr}(X)=\frac{1}{2 \pi} \int_{0}^{2 \pi} U_{\rho *}(X) d \rho
$$

is linear and tame. Therefore $T_{e} D i f f^{U(1)}(M)$ is a tame direct summand of the tame Frechet space $T_{e} \operatorname{Dif} f(M)$. It follows that $T_{e} D i f f^{U(1)}(M)$ is also a tame Frechet space. Given a smooth vector field $X$ on $M$, we denote $\exp _{p} X$ the time $=1$ point of the geodesic (with respect to the invariant metric $g$ ) passing through $p$ in $M$ with the velocity $X$. Define $\Psi: T_{e} \operatorname{Diff}(M) \rightarrow$ $\operatorname{Diff}(M)$ by $\Psi(X)(p)=\exp _{p} X$. It is known that $\Psi$ parametrizes $\operatorname{Diff}(M)$ near $e$. Moreover, $\Psi$ maps the subspace $T_{e} D i f f^{U(1)}(M)$ (injectively for sure) 
into $\operatorname{Dif} f^{U(1)}(M)$ since $U_{\rho}$ 's are isometries with respect to $g$. We claim that $\Psi$ restricted to $T_{e} D i f f^{U(1)}(M)$ is actually surjective onto $\operatorname{Dif} f^{U(1)}(M)$. Suppose $\phi$ is a $U(1)$-equivariant diffeomorphism near $e$ and let $X=\Psi^{-1}(\phi)$. We need to show that $X$ is $U(1)$-invariant. Let $\phi_{t}$ denote the geodesic flow of $X$ with respect to $g$. Since $U_{\rho}$ is an isometry (with respect to $g$ ), $U_{\rho} \circ \phi_{t}(p)$ is a geodesic connecting $U_{\rho}(p)(t=0)$ and $U_{\rho} \circ \phi(p)(t=1)$ for $p$ in $M$. On the other hand, $\phi_{t} \circ U_{\rho}(p)$ is a geodesic connecting $U_{\rho}(p)(t=0)$ and $\phi \circ U_{\rho}(p)(t=1)$ which equals $U_{\rho} \circ \phi(p)$ by the assumption. Now for $\phi$ close enough to $e$, the uniqueness of geodesics connecting two points in a convex neighborhood implies $U_{\rho} \circ \phi_{t}=\phi_{t} \circ U_{\rho}$. It follows that $U_{\rho *} \circ X=X \circ U_{\rho}$, i.e. $X$ is $U(1)$-invariant, so $\Psi$ parametrizes $\operatorname{Diff}^{U(1)}(M)$ as a tame Frechet submanifold of $\operatorname{Diff}(M)$ near $e$. For a $U(1)$-equivariant diffeomorphism $\psi \neq e$, the local parametrization $\Psi_{\psi}$ defined by $\Psi_{\psi}(X)=\Psi(X) \circ \psi$ for $\operatorname{Diff}(M)$ also parametrizes $\operatorname{Diff}^{U(1)}(M)$ near $\psi$ when $X$ 's are restricted to $T_{e} \operatorname{Diff}^{U(1)}(M)$. We have shown that $\operatorname{Diff}^{U(1)}(M)$ is a tame Frechet submanifold of $\operatorname{Diff}(M)$. It is easy to see that $\operatorname{Diff} f^{U(1)}(M)$ is a group under composition and $\operatorname{Diff}(M)$ is a smooth tame Lie group (p.148 in [Ha]). It follows that $\operatorname{Diff}^{U(1)}(M)$ is also a smooth tame Lie group.

Proof of Theorem 5.1. The action $P: \operatorname{Diff} f^{U(1)}(M) \times \mathfrak{B}^{U(1)} \rightarrow \mathfrak{B}^{U(1)}$ is described by

$$
P(\phi, \theta)=\phi^{*} \theta /\left|\phi^{*} \theta\right|_{g}
$$

It is easy to see that the maps: $(\phi, \theta) \rightarrow \phi^{*} \theta$ and $\eta \rightarrow\langle\eta, \eta\rangle_{g}^{\frac{1}{2}}=|\eta|_{g}$ are smooth tame, so $P$ is smooth tame. Let $b_{\theta}(X)=D_{1} P(e, \theta)(X)$, the partial derivative with respect to the first variable of $P$ at the identity $e$. Let $H$ denote the contact bundle annihilated by the contact form $\theta$. Let $\phi_{t}$ be a smooth family of $U(1)$-equivariant diffeomorphisms such that $\phi_{0}=e$ and $\left.\frac{d}{d t}\right|_{t=0} \phi_{t}=X$. We compute

$$
\begin{aligned}
\left.\frac{d}{d t}\right|_{t=0}\left|\phi_{t}^{*} \theta\right|_{g}^{-1} & =\left.\frac{d}{d t}\right|_{t=0}\left(\left|\phi_{t}^{*} \theta\right|_{g}^{2}\right)^{\frac{-1}{2}} \\
& \left.=\left(-\frac{1}{2}\right) \cdot 2\langle X\rfloor d \theta, \theta\right\rangle_{g} \\
& =-\langle X\rfloor d \theta, \theta\rangle_{g}
\end{aligned}
$$


for $X$ tangent to $H$. It follows that for $X$ tangent to $H$,

$$
\begin{aligned}
b_{\theta}(X) & =\left.\frac{d}{d t}\right|_{t=0} P\left(\phi_{t}, \theta\right) \\
& =\left.\frac{d}{d t}\right|_{t=0} \phi_{t}^{*} \theta+\left.\frac{d}{d t}\right|_{t=0}\left|\phi_{t}^{*} \theta\right|_{g}^{-1} \theta \\
& =X\rfloor d \theta-\langle X\rfloor d \theta, \theta\rangle_{g} \theta \\
& \left.=\pi_{H^{*}}(X\rfloor d \theta\right) .
\end{aligned}
$$

Here $\pi_{H^{*}}$ is the projection onto the orthogonal complement of $\theta$. Now given $\eta$ in $T_{\theta} \mathfrak{B}^{U(1)}$, we want to find $X$ such that $\left.\pi_{H^{*}}(X\rfloor d \theta\right)=\eta$. It is easy to find a unique $X$ tangent to $H$ so that $X\rfloor d \theta=\eta$ on $H$. Since $\theta, H$, and $\eta$ are all $U(1)$-invariant, it follows that $X$ is also $U(1)$-invariant by uniqueness. On the other hand, $\left.X\rfloor d \theta=\pi_{H^{*}}(X\rfloor d \theta\right)$ on $H$. But $\eta$ is orthogonal to $\theta$. Thus $\left.b_{\theta}(X)=\pi_{H^{*}}(X\rfloor d \theta\right)=\eta$. We have proved that the map: $X \rightarrow b_{\theta}(X)=D_{1} P(e, \theta)(X)$ from $T_{e} D i f f^{U(1)}(M)$ to $T_{\theta} \mathfrak{B}^{U(1)}$ is surjective with a right inverse $\eta \rightarrow X$. It is easy to check that the linear map: $\eta \rightarrow X$ is tame. Now our theorem follows from Theorem 2.4.1 on p.198 in [Ha].

Proof of lemma 5.3. First observe that in the proof of Theorem 2.4.1 on p.198 in [Ha], we actually show that the action with a reference point fixed is locally surjective and has a smooth tame right inverse by Theorem 1.1.3 on p.172 in [Ha]. This means in our case the action $\phi \rightarrow P\left(\phi, \theta_{0}\right)$ with $\theta_{0}$ fixed is locally surjective and has a smooth tame right inverse $V$. Set $s(\widetilde{H})=(V(\widetilde{\theta}))^{-1}$ where $\widetilde{\theta}$ is the contact form associated to the contact bundle $\widetilde{H}$ near $H_{0}$.

\section{Appendix B: An infinitesimal slice of $\widetilde{\mathfrak{M}}_{-1,0} / C_{\hat{H}}$.}

Take a family of pseudohermitian structures $\left(J_{(t)}, \theta_{(t)}\right)$ on $(\hat{S}, \hat{H})$ with $J_{(0)}=$ $\hat{J}, \theta_{(0)}=\hat{\theta}$. At $t=0$, express

$$
\left(\dot{J}_{(t)}, \dot{\theta}_{(t)}\right)=\left(2 E_{1}{ }^{\overline{1}} \hat{\theta}^{1} \otimes \hat{Z}_{\overline{1}}+2 E_{\overline{1}}^{1} \hat{\theta}^{\overline{1}} \otimes \hat{Z}_{1}, 2 h \hat{\theta}\right)
$$

where $E_{1}{ }^{\overline{1}}$ is a deformation tensor at $\hat{J}$ and $h$ is just a real-valued function. (see (2.14) on p.231 in [CL1]; also note $\theta_{(t)} \mid \hat{H}=0$ ) Next we observe the action of $C_{\hat{H}}$. Let $\phi_{t} \in C_{\hat{H}}$ be a family of contact diffeomorphisms with 
$\phi_{0}=$ identity. Compute

$$
\begin{aligned}
\left.\frac{d}{d t}\right|_{t=0}\left(\phi_{t}^{*} \hat{J}, \phi_{t}^{*} \hat{\theta}\right) & =\left(L_{X_{f}} \hat{J}, L_{X_{f}} \hat{\theta}\right) \quad(\text { Lemma } 3.4 \text { on p.239 in [CL1] }) \\
& =\left(2 B_{\hat{J}}^{\prime} f,-(\hat{T} f) \hat{\theta}\right)
\end{aligned}
$$

((3.13) and the proof of Lemma 3.4 in [CL1])

where $B_{j}^{\prime}$ is the second-order operator defined on p.236 in [CL1] and $\hat{T}$ is the vector field uniquely determined by $\hat{\theta}(\hat{T})=1, \hat{T}\lrcorner d \hat{\theta}=0$. Define

$$
\widetilde{B}_{\hat{J}}^{\prime} f=B_{\hat{J}}^{\prime} f-\frac{1}{2}(\hat{T} f) \hat{\theta} .
$$

Then we have the following orthogonal decomposition:

$$
T_{(\hat{J}, \hat{\theta})}\{(J, \theta): \theta \mid \hat{H}=0\}=\operatorname{Ker} \widetilde{B}_{\hat{J}} \oplus \text { Range } \widetilde{B}_{\hat{J}}^{\prime}
$$

where $\widetilde{B}_{\hat{J}}$ is the adjoint operator of $\widetilde{B}_{\hat{J}}^{\prime}$, given by

$$
\widetilde{B}_{\hat{J}}(\widetilde{E})=B_{\hat{J}} E+\frac{1}{2} h_{, o}
$$

for $\widetilde{E}=E+h \hat{\theta}, E=E_{1}{ }^{\overline{1}} \hat{\theta}^{1} \otimes \hat{Z}_{\overline{1}}+E_{\overline{1}}^{1} \hat{\theta}^{\overline{1}} \otimes \hat{Z}_{1}$. Here $B_{\hat{J}}$ is defined on p.235 in [CL1]. Note that Range $\widetilde{B}_{\hat{J}}^{\prime}$ is the tangent space of the orbit of $C_{\hat{H}}$ passing through $(\hat{J}, \hat{\theta})$. The decomposition (B.2) is valid either in $L^{2}$ category or in $C^{\infty}$ category mainly because of the fourth-order operator $\widetilde{B}_{\hat{J}} \widetilde{B}_{\hat{J}}^{\prime}=\Delta_{\hat{J}}-\frac{1}{4} \hat{T}^{2}=\frac{1}{2} \mathfrak{L}_{\alpha}^{*} \mathfrak{L}_{\alpha}+O_{2}\left(\alpha=i \sqrt{\frac{7}{2}}\right)$ being subelliptic. (see Lemma 2.1 in [CL2]) Now linearizing the equations $R_{J, \theta} \equiv-1, A_{J, \theta} \equiv 0$ at $(\hat{J}, \hat{\theta})$ in the direction $(\cdot \rightarrow J, \cdot \rightarrow \theta)$ given by (B.1), we obtain

$$
\left\{\begin{array}{l}
i\left(E_{1}{ }^{\overline{1}}{ }^{1}{ }_{1}-E_{\overline{1}^{1}, 1}{ }^{\overline{1}}\right)+2 h+4 \Delta_{b} h=0 \\
E_{\overline{1}^{1}, 0}{ }^{1}+2 h_{, \overline{1}}{ }^{1}=0
\end{array}\right.
$$

by (5.15), (5.9) in [Lee] and (2.20), (2.18) in [CL1], where $\Delta_{b} h=-\left(h_{, 1}{ }^{1}+\right.$ $\left.h_{, 1}{ }^{\overline{1}}\right)$. (see (4.10) in [Lee]) Since elements in Range $\widetilde{B}_{\hat{J}}^{\prime}$ satisfy the linear equations (B.3), we get from (B.2) that an infinitesimal slice of $\widetilde{\mathfrak{M}}_{-1,0} / C_{\hat{H}}$ is the intersection of $\operatorname{Ker} \widetilde{B}_{\hat{J}}$ and the solution space of (B.3). Write $\widetilde{K}=K+k \theta$ 
in this infinitesimal slice. It follows that $\widetilde{K}$ satisfies the following system of equations:

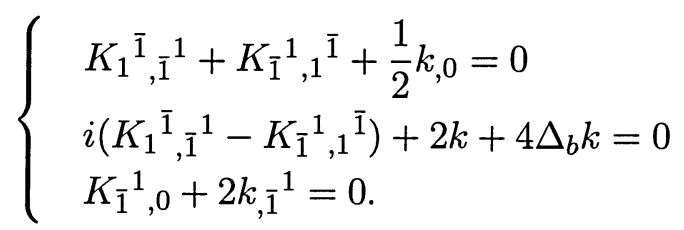

\section{References.}

[Au] T. Aubin, Nonlinear analysis on manifolds. Monge-Ampere equations. G.M.W. 252 (1982), Springer-Verlag.

[BD] J. Bland and T. Duchamp, The group of contact diffeomorphisms for compact contact manifolds, preprint.

[BS] D. Burns and S. Shnider, Spherical hypersurfaces in complex manifolds, Invent. Math. 33 (1976), 223-246.

[Cap] L. Caporaso, A compactification of the universal Picard variety over the moduli space of stable curves, J. Amer. Math. Soc. 7 (1994), 589-660.

[Car] E. Cartan, Sur la geometrie pseudo-conforme des hypersurfaces de deux variables complexes I, II, 1217-1238, Oeuvres II, 2, 1231-1304, III, 2.

[CH] S.-S. Chern and R. S. Hamilton, On Riemannian metrics adapted to threedimensional contact manifolds, Lect. Notes 1111, Springer-Verlag, 279-308.

[CL1] J.-H. Cheng and J. M. Lee, The Burns-Epstein invariant and deformation of CR structures, Duke Math. J. 60 (1990), 221-254.

[CL2] A local slice theorem for 3-dimensional CR structures, Amer. J. Math. 117 (1995), 1249-1298.

[CM] S.-S. Chern and J. K. Moser, Real hypersurfaces in complex manifolds, Acta Math. 133 (1974), 219-271.

[Ds] C. D'Souza, Compactification of generalized Jacobian, Proc. Indian Acad. Sci. Sect. A, Math. Sci. 88 (1979), 419-457.

[Eb] D. Ebin, The manifold of Riemannian metrics, AMS Proc. on global analysis, Berkeley 1968, 11-40.

[ENS] F. Ehlers, W. D. Neumann, and J. Scherk, Links of surface singularities and CR space forms, Comment. Math. Helvetici, 62 (1987), 240-264.

[Ep] C. Epstein, CR-structures on three dimensional circle bundles, Invent. math. 109 (1992), 351-403. 
[FG] E. Falbel and N. Gusevskii, Spherical CR-Manifolds of Dimension 3, Bol. Soc. Bras. Mat. 25 (1994), 31-56.

[Fo] G. B. Folland, Subelliptic estimates and function spaces on nilpotent Lie groups, Ark. Mat. 13 (1975), 161-207.

[FK] G. B. Folland and J. J. Kohn, The Neumann problem for the CauchyRiemann complex, Annals Math. Studies, no. 75, Princeton Univ. Press, Princeton, 1972.

[FS] G. B. Folland and E. M. Stein, Estimates for the $\bar{\partial}_{b}$ complex and analysis on the Heisenberg group, Commun. Pure and Applied Math. 27 (1974), $429-522$.

[Go] W. M. Goldman, Representations of fundamental groups of surfaces, Proceedings of Geometry and Topology, University of Maryland, 1983-1984 Springer Lecture Notes 1167, 1985, 95-117.

[Gr] J. W. Gray, Some global properties of contact structures, Ann. Math. 69 (1959), 421-450.

[GT] D. Gilbarg and N. S. Trudinger, Elliptic partial differential equations of second order, G.M.W. 224 (1983), Springer-Verlag.

[Ha] R. S. Hamilton, The inverse function theorem of Nash and Moser, Bull. Amer. Math. Soc. 7 (1982), 65-222.

[HL] R. Harvey and B. Lawson, On boundaries of complex analytic varieties I, Ann. Math. 102 (1975), 233-290.

[Is] M. Ishida, Compactifications of a family of generalized Jacobian varieties, Proc. Internat. Symp. on Alg. Geom. (Kyoto 1977), Kinokuniya Bookstore, Tokyo 1978, 503-524.

[JL] D. Jerison and J. M. Lee, The Yamabe problem on CR manifolds, J. Diff. Geom. 25 (1987), 167-197.

[K1] J. J. Kohn, The range of the tangential Cauchy-Riemann operator, Duke Math. J. 53 (1986), 525-545.

[K2] Estimates for $\bar{\partial}_{b}$ on pseudo-convex $C R$ manifolds, Proc. Symp. Pure Math. 43 (1985), 207-217.

[Ko] S. Kobayashi, Hyperbolic manifolds and holomorphic mappings, Pure and Applied Math. 2, 1970, Marcel Dekker, New York.

[La] S. Lang, Differential manifolds, 1972. 
[Lee] J. M. Lee, The Fefferman metric and pseudohermitian invariants, Trans. AMS, 296 (1986), 411-429.

[NN] A. Newlander and L. Nirenberg, Complex analytic coordinates in almostcomplex manifolds, Ann Math. 65 (1957), 391-404.

[OS] T. Oda and C. Seshadri, Compactifications of the generalized Jacobian variety, Trans. Amer. Math. Soc. 253 (1979), 1-90.

[Pa] P. Pansu, Compactness, Progress in Math. 117 (ed. Audin and Lafontaine): Holomorphic curves in symplectic geometry.

[Ra] M. S. Raghunathan, Discrete subgroups of Lie groups, Erg. der Math. Bd. 68, 1972, Springer-Verlag.

[SS] M. Seppala and T. Sorvali, Geometry of Riemann surfaces and Teichmuller spaces, 1992, North-Holland.

[Tan] N. Tanaka, A Differential Geometric Study on Strongly Pseudo-Convex Manifolds, 1975, Kinokuniya Co. Ltd., Tokyo.

[Tr] A. J. Tromba, Teichmuller theory in Riemannian geometry, 1992, Birkhauser.

[We1] S. M. Webster, Pseudohermitian structures on a real hypersurface, J. Diff. Geom. 13 (1978), 25-41.

[We2] On the pseudo-conformal geometry of a Kahler manifold, Math. Z. 157 (1977), 265-270.

[Y] S. S.-T. Yau, Kohn-Rossi cohomology and its application to the complex Plateau problem I, Ann. Math. 113 (1981), 67-110.

RECEIVED APRIL 2, 1998.

Academia Sinica, Nankang

TAIPEI, TAIWAN, R.O.C.

AND

National TAIWAN UNIVERSITY

TAIPEI, TAIWAN, R.O.C.

E-mail addresses: Cheng@math.sinica.edu.tw

ihtsai@math.ntu.edu.tw 